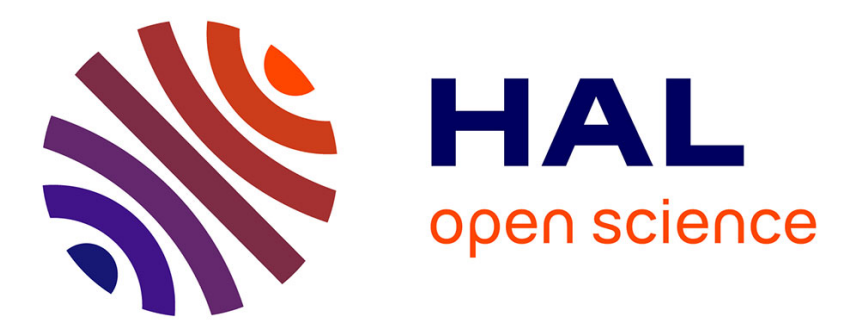

\title{
Inhomogeneity and anisotropy effects on the redistribution term in RANS modelling
}

Remi Manceau, Meng Wang, Dominique R. Laurence

\section{To cite this version:}

Remi Manceau, Meng Wang, Dominique R. Laurence. Inhomogeneity and anisotropy effects on the redistribution term in RANS modelling. Journal of Fluid Mechanics, 2001, 438, pp.307-338. 10.1017/S0022112001004451 . hal-02990731

\section{HAL Id: hal-02990731 \\ https://hal.science/hal-02990731}

Submitted on 5 Nov 2020

HAL is a multi-disciplinary open access archive for the deposit and dissemination of scientific research documents, whether they are published or not. The documents may come from teaching and research institutions in France or abroad, or from public or private research centers.
L'archive ouverte pluridisciplinaire HAL, est destinée au dépôt et à la diffusion de documents scientifiques de niveau recherche, publiés ou non, émanant des établissements d'enseignement et de recherche français ou étrangers, des laboratoires publics ou privés.

\section{(ㅇ)(1) $\$$}

Distributed under a Creative Commons Attribution - NonCommercial - NoDerivatives 44.0 


\title{
Inhomogeneity and anisotropy effects on the redistribution term in RANS modelling
}

\author{
By RÉMI MANCEA U ${ }^{1} \dagger, M E N G$ WA N G \\ AND DOMINIQUE LAURENCE I $^{1,3}$ \\ ${ }^{1}$ Laboratoire National d'Hydraulique, Électricité de France, 78401 Chatou, France \\ ${ }^{2}$ Center for Turbulence Research, BLDG. 500, Stanford University, Stanford, CA 94305-3030, \\ USA \\ ${ }^{3}$ Department of Mechanical Engineering, UMIST, George Begg Building, Sackville Street, \\ Manchester, M60 1QD, UK
}

(Received ?? and in revised form ??)

A channel flow DNS database at $R e_{\tau}=590$ is used to assess the validity of modelling the redistribution term in the Reynolds stress transport equations by elliptic relaxation. The model assumptions are found to be globally consistent with the data. However, the correlation function between the fluctuating velocity and the Laplacian of the pressure gradient, which enters the integral equation of the redistribution term, is shown to be anisotropic. It is elongated in the streamwise direction and strongly asymmetric in the direction normal to the wall, in contrast to the isotropic, exponential model representation used in the original elliptic relaxation model. This discrepancy is the main cause for the slight amplification of the energy redistribution in the log layer as predicted by the elliptic relaxation equation. New formulations of the model are proposed in order to correct this spurious behaviour, by accounting for the rapid variations of the length scale and the asymmetrical shape of the correlation function. These formulations do not rely on the use of wall echo correction terms to damp the redistribution. The belief that the damping is due to the so called "wall echo" effect is called into question through the present DNS analysis.

\section{Introduction}

In second moment closures, one of the most important and difficult tasks is to model the pressure gradient-velocity correlation in the Reynolds stress transport equations. Indeed, since the production does not need any modelling at this closure level, particular attention must be focused on this correlation term and on the dissipation. In a channel flow (e.g. Mansour, Kim \& Moin 1988) the pressure gradient-velocity correlation, whose effect is mainly to redistribute the energy among the Reynolds stresses (hence called the "redistribution term"), is the only source term in the budgets of the wall-normal and spanwise Reynolds stresses; it balances the production in the shear stress budget.

Since the pioneering works of Chou (1945) and Rotta (1951), the local approach, which algebraically relates the unclosed redistribution term to the Reynolds stress anisotropy, mean strain, and mean vorticity tensors, has been popular in the turbulence modelling community. All standard models are based on this approach. The redistribution term is

$\dagger$ Present address: Laboratoire d'Études Aérodynamiques, UMR CNRS 6609, SP2MI, Téléport 2, Bd Pierre et Marie Curie, BP 30179, 86962 Futuroscope, Poitiers, France 
written in an integral form and split into three parts, the rapid, slow and surface parts. The slow part, which does not involve any mean flow quantity, is modelled in terms of the Reynolds stress anisotropy. The rapid part is expressed in terms of products between mean velocity gradients and a fourth-order tensor, based on the assumption that the mean velocity gradient is locally constant. This quasi-homogeneous approach has been the starting point of almost all second moment closure models. In most of them, linear ones in particular, (e.g. Rotta 1951; Naot, Shavit \& Wolfshtein 1973; Launder, Reece \& Rodi 1975) and even fully nonlinear ones (e.g. Fu, Launder \& Tselepidakis 1987), the surface part is neglected or modelled by wall echo terms, as suggested by Gibson \& Launder (1978). In others, the influence of the solid boundary is accounted for through variable coefficients, leading to quasi-linear models, such as that of Speziale, Sarkar \& Gatski (1991). In the recent model of Craft \& Launder (1996), the nonlinear formulation directly includes wall induced effects.

However, the validity of the quasi-homogeneous approximation used for the rapid part is questionable. It assumes that the mean velocity gradient varies sufficiently slowly to allow it to be taken outside the integral, which is not the case in strongly inhomogeneous turbulence. Bradshaw, Mansour \& Piomelli (1987) used the channel flow DNS of Mansour et al. (1988) to show that this hypothesis is correct down to $y^{+}=40$, but totally invalid below this value. Another weakness of the quasi-homogeneous approach is the loss of the non-local character of the redistribution term. The integral equation for the latter, which involves two-point correlations between velocities and Laplacian of the pressure gradient, shows that it actually depends on the mean flow and the turbulence state at all points of the domain. Kim (1989) showed that in a channel, except in the very near-wall region, the redistribution term takes contributions from all the domain, including the opposite wall. Furthermore, a number of theoretical studies (e.g. Hunt \& Graham 1978) as well as direct numerical simulations (Perot \& Moin 1993) showed that the structures of the flow, and the associated length scales, are strongly affected by the presence of a solid boundary even in the absence of mean shear, because of the blocking effect which is non-local. In particular, the two-point correlations of the wall-normal velocity are, as shown by Hunt et al. (1989), influenced near the wall by the image eddies. These non-local effects make the redistribution term difficult, if not impossible, to model in terms of local variables.

Furthermore, the quasi-homogeneous models cannot in general be integrated down to solid boundaries without introducing corrections, such as damping functions (there are exceptions, such as the Craft \& Launder 1996 model). Damping functions are not universal, since they are derived by fitting experimental or DNS results with little theoretical justification.

In order to avoid such problems, Durbin $(1991 ; 1993)$ introduced a novel approach. He proposed to model directly the two-point correlation in the integral equation of the redistribution term, using an isotropic, exponential function. A convolution product is obtained, which can be inverted to give the so-called elliptic relaxation approach. The redistribution term is no longer given by an algebraic relation, but rather by a differential equation. The non-local character is preserved through the elliptic operator $\left(1-L^{2} \nabla^{2}\right)$, and the model can be integrated down to the wall. A notable feature of this approach is that the source term of the elliptic relaxation equation can be given by any quasi-homogeneous model. Hence, it enables the derivation of models valid down to solid boundaries, from the quasi-homogeneous models cited above, which have been tested over a wide range of different flows. Even though some intuitive assumptions have been made, Durbin's model is based on a theoretical approach, leading to the hope that it is somewhat universal, unlike damping functions.

The elliptic relaxation model has led to very encouraging results, especially as applied 
to the $\overline{v^{2}}-f$ (or $k-\varepsilon-\overline{v^{2}}$ ) model, which is a version of the full Reynolds stress model reduced to three transport equations. Successful predictions include, but are not limited to, flows with adverse pressure gradient and around bluff bodies (Durbin 1995), three dimensional boundary layers (Parneix, Durbin \& Behnia 1998), aerodynamics (Lien, Durbin \& Parneix 1997), and heat transfer (Behnia, Parneix \& Durbin 1998; Manceau, Parneix \& Laurence 2000).

Despite the remarkable success, rooms for improving the elliptic relaxation model exist. Many of the underlying model assumptions, introduced intuitively, have not been validated by either experiments or DNS. The objective of the present study is to evaluate these assumptions through the analysis of a channel flow DNS database, and to find ways to improve the theoretical basis and performance of the model. The main issues to be examined include the validity of the two-point correlation approximation employed by Durbin (1991), the validity of the length scale used in the elliptic operator, and the unsatisfactory behaviour of the model in the logarithmic layer. A full explanation of these issues is given in $\S 2$ and $\S 3$. In $\S 4$, the results of the DNS analysis are discussed. It is found that the elliptic relaxation model is globally consistent with the simulation data, and that the correlation length scale is adequately modelled by the turbulent length scale bounded near the wall by the Kolmogorov length scale. However, the correlation function between the fluctuating velocity and the Laplacian of the pressure gradient is strongly anisotropic and inhomogeneous. Its approximation by an isotropic, exponential function is responsible for the spurious amplification of the energy redistribution in the log layer, as predicted by the model. It is further discovered that the so called "wall echo" effect increases the redistribution of energy, contrary to the general belief. The physical insights gained through the DNS study are used, in $\S 5$, to develop new formulations of the model that rectify the erroneous logarithmic-layer behaviour. This is achieved by taking into account the influence of strong inhomogeneity and anisotropy on the redistribution term, using a spatially variable length scale and an asymmetric model of the correlation function. Unlike some previous ad hoc formulations, the new formulations emphasize a systematic, scientific approach to turbulence modelling, guided by the DNS data. Finally, $\S 6$ summarizes the major findings and accomplishments of this work.

\section{Theoretical background}

\subsection{Integral equation of the redistribution term}

The pressure gradient-velocity correlation entering the Reynolds stress transport equations is

$$
\rho \phi_{i j}^{*}=-\overline{u_{i} \frac{\partial p}{\partial x_{j}}}-\overline{u_{j} \frac{\partial p}{\partial x_{i}}}
$$

where $\rho$ is the density, $p$ is the fluctuating pressure and $u_{i}$ are the fluctuating velocity components. The overline indicates ensemble average. Traditionally, this term is split into pressure-strain correlation and pressure diffusion. However, since this splitting is nonunique (Lumley 1975) and inconsistent with the Navier-Stokes equations in the limit of two-dimensional turbulence (Speziale 1985), it appears more appropriate to model the pressure gradient-velocity correlation as a whole. Since the pressure diffusion is negligible in the main part of the flow, $\phi_{i j}^{*}$ can be regarded as the energy redistribution between the components of the Reynolds stress, except in the near-wall region, where it balances the difference between dissipation and molecular diffusion.

The gradient of the pressure fluctuation is the solution of the Poisson equation obtained 
from the divergence of the fluctuating part of the Navier-Stokes equations,

$$
\nabla^{2} \frac{\partial p}{\partial x_{k}}=-\rho \frac{\partial}{\partial x_{k}}\left(2 \frac{\partial U_{i}}{\partial x_{j}} \frac{\partial u_{j}}{\partial x_{i}}+\frac{\partial u_{i}}{\partial x_{j}} \frac{\partial u_{j}}{\partial x_{i}}-\overline{\frac{\partial u_{i}}{\partial x_{j}} \frac{\partial u_{j}}{\partial x_{i}}}\right) .
$$

Following Kim (1989), it will be assumed that the contribution from the inhomogeneous boundary condition, or the "Stokes part", is negligible. Accordingly, $\partial p / \partial x_{k}$ approximately satisfies a homogeneous Neumann boundary condition.

Using the Green function $G_{\Omega}$ of the domain, the solution of (2.2) takes the form

$$
\frac{\partial p}{\partial x_{k}}(\boldsymbol{x})=\int_{\Omega} \nabla^{2} \frac{\partial p}{\partial x_{k}}\left(\boldsymbol{x}^{\prime}\right) G_{\Omega}\left(\boldsymbol{x}, \boldsymbol{x}^{\prime}\right) \mathrm{d} V\left(\boldsymbol{x}^{\prime}\right),
$$

where $\boldsymbol{x}$ and $\boldsymbol{x}^{\prime}$ denote position vectors, and $\mathrm{d} V$ the elementary volume. The integral equation of the redistribution term can be derived from (2.1) and (2.3):

$$
\rho \phi_{i j}^{*}(\boldsymbol{x})=\int_{\Omega} \Psi_{i j}\left(\boldsymbol{x}, \boldsymbol{x}^{\prime}\right) G_{\Omega}\left(\boldsymbol{x}, \boldsymbol{x}^{\prime}\right) \mathrm{d} V\left(\boldsymbol{x}^{\prime}\right),
$$

where $\Psi_{i j}\left(\boldsymbol{x}, \boldsymbol{x}^{\prime}\right)$ denotes the two-point correlation between the velocity and the Laplacian of the pressure gradient:

$$
\Psi_{i j}\left(\boldsymbol{x}, \boldsymbol{x}^{\prime}\right)=\overline{-u_{i}(\boldsymbol{x}) \nabla^{2} \frac{\partial p}{\partial x_{j}}\left(\boldsymbol{x}^{\prime}\right)}-\overline{u_{j}(\boldsymbol{x}) \nabla^{2} \frac{\partial p}{\partial x_{i}}\left(\boldsymbol{x}^{\prime}\right)} .
$$

\subsection{The elliptic relaxation equation}

In (2.4), the two-point correlations between the velocity and the Laplacian of the pressure gradient need to be modelled. Durbin (1991) defined a correlation function

$$
\Psi_{i j}\left(\boldsymbol{x}, \boldsymbol{x}^{\prime}\right)=\Psi_{i j}\left(\boldsymbol{x}^{\prime}, \boldsymbol{x}^{\prime}\right) f\left(\boldsymbol{x}, \boldsymbol{x}^{\prime}\right),
$$

and modelled it by

$$
f\left(\boldsymbol{x}, \boldsymbol{x}^{\prime}\right)=\exp \left(-\frac{r}{L}\right),
$$

where $r=\left\|\boldsymbol{x}^{\prime}-\boldsymbol{x}\right\|$ and $L$ is the correlation length scale. This approximation is the corner-stone of the elliptic relaxation model and the validity of (2.7) is the main concern of this paper.

In a free space, using the model (2.7), the redistribution term can be written as

$$
\rho \phi_{i j}^{*}(\boldsymbol{x})=-\int_{\Omega} \Psi_{i j}\left(\boldsymbol{x}^{\prime}, \boldsymbol{x}^{\prime}\right) \underbrace{\frac{\exp \left(-\frac{r}{L}\right)}{4 \pi r}}_{E(r)} \mathrm{d} V\left(\boldsymbol{x}^{\prime}\right) .
$$

In this form, $\phi_{i j}^{*}$ appears as a convolution product between $\Psi_{i j}$ and $E(r)$, which is the free-space Green function associated with the operator $-\nabla^{2}+1 / L^{2}$. Due to (2.6), the one-point correlation in the integrand is expressed as a function of $\boldsymbol{x}^{\prime}$. If it were expressed as a function of $\boldsymbol{x}$, the one-point correlation could have been taken outside the integral in (2.8), and the non-local effect would have been lost or entirely recast into $f\left(\boldsymbol{x}, \boldsymbol{x}^{\prime}\right)$, which would then be more difficult to model. The convolution integral (2.8) can be inverted, yielding the elliptic relaxation equation:

$$
\left.\phi_{i j}^{*}-L^{2} \nabla^{2} \phi_{i j}^{*}=-\frac{L^{2}}{\rho} \overline{\left(u_{i} \nabla^{2} \frac{\partial p}{\partial x_{j}}\right.}+\overline{u_{j} \nabla^{2} \frac{\partial p}{\partial x_{i}}}\right) .
$$

In homogeneous situations, the second term on the left hand side of this equation van- 
ishes. Therefore, Durbin (1991) proposed to replace the right hand side by any quasihomogeneous model $\phi_{i j}^{h}$, which leads to the model

$$
\phi_{i j}^{*}-L^{2} \nabla^{2} \phi_{i j}^{*}=\phi_{i j}^{h}
$$

This method provides a simple way of extending quasi-homogeneous models down to solid boundaries, when appropriate boundary conditions for $\phi_{i j}^{*}$ are applied (Durbin 1993).

\section{Presentation of the DNS assessment}

\subsection{Issues to examine}

The elliptic relaxation approach is mainly based on the assumption that the correlation function $f\left(\boldsymbol{x}, \boldsymbol{x}^{\prime}\right)$, defined by (2.6), can be modelled by an exponential function. This approximation was introduced by Durbin (1991) on an intuitive basis, in order to preserve the non-local effect on the redistribution term. However, its validity has never been checked before, and the shape of $\Psi_{i j}\left(\boldsymbol{x}, \boldsymbol{x}^{\prime}\right)$ needs to be investigated. The DNS database of the channel flow at $R e_{\tau}=590$ (Moser, Kim \& Mansour, 1999) will be used for this purpose.

Another aim of this work is to evaluate the correlation length scale involved in the model (2.7) for the correlation function $f\left(\boldsymbol{x}, \boldsymbol{x}^{\prime}\right)$. If the turbulent length scale were used in the whole flow, since it goes to zero at solid boundaries, the elliptic operator $L^{2} \nabla^{2}$ would vanish at the wall, introducing a singularity in the differential equation. Therefore, Durbin (1991) proposed using the standard turbulent length scale in the main part of the flow, and the Kolmogorov length scale in the vicinity of the wall, i.e.,

$$
L=C_{L} \max \left(C_{\eta} \frac{\nu^{3 / 4}}{\varepsilon^{1 / 4}} ; \frac{k^{3 / 2}}{\varepsilon}\right) .
$$

It is of interest to evaluate precisely the correlation length scale from the DNS data, in order to assess the validity of (3.1).

The ultimate objective of this work is to find ways to improve the model. As pointed out by Wizman et al. (1996), the elliptic operator does not behave entirely correctly in the logarithmic layer. Suppose, for instance, that the Isotropisation of Production model (Naot, Shavit \& Wolfshtein 1973; Launder, Reece \& Rodi 1975), denoted henceforth as IP model, and the Rotta (1951) model are used as the rapid and slow parts of the source term $\phi_{i j}^{h}$ in (2.10). The redistribution term is then given by

$$
\phi_{i j}^{*}-L^{2} \nabla^{2} \phi_{i j}^{*}=-C_{1} \frac{\varepsilon}{k}\left(\overline{u_{i} u_{j}}-\frac{2}{3} k \delta_{i j}\right)-C_{2}\left(P_{i j}-\frac{2}{3} P \delta_{i j}\right),
$$

where $P_{i j}=-\overline{u_{i} u_{k}} \partial U_{j} / \partial x_{k}-\overline{u_{j} u_{k}} \partial U_{i} / \partial x_{k}$ and $P=\frac{1}{2} P_{i i}$. In the logarithmic layer, the Reynolds stresses are constant, and the production and the dissipation behave as $y^{-1}$. Thus, the right hand side in (3.2) behaves as $y^{-1}$, and the redistribution term is then given by $\phi_{i j}^{*} \approx 1.51 \phi_{i j}^{h}$. This result shows that the elliptic operator leads to an amplification of the redistribution. Note that the same amplification occurs with any model for $\phi_{i j}^{h}$.

The overestimation of the energy redistribution by the Rotta \& IP model in the logarithmic layer has led a number of modellers to introduce wall echo type terms, following Gibson \& Launder (1978). It would be desirable for the elliptic relaxation equation to compensate for this shortcoming. Some models, such as the Speziale, Sarkar \& Gatski (1991) model, or Craft \& Launder (1996) model, correctly reproduce the redistribution in the logarithmic layer. In this case, it would be preferable that the elliptic relaxation model be neutral, producing neither amplification nor reduction of the redistribution. 
Based on the above considerations, Wizman et al. (1996) proposed two new formulations of the elliptic relaxation equation. First, they introduced a neutral formulation by taking $L^{2}$ in (2.10) inside the Laplacian operator:

$$
\phi_{i j}^{*}-\nabla^{2}\left(L^{2} \phi_{i j}^{*}\right)=\phi_{i j}^{h} .
$$

Secondly, for models that overestimate the redistribution, they proposed

$$
\phi_{i j}^{*}-L^{2} \nabla \cdot\left(\frac{1}{L^{2}} \nabla\left(L^{2} \phi_{i j}^{*}\right)\right)=\phi_{i j}^{h},
$$

which exhibits the expected damping. Laurence \& Durbin (1994) and Durbin \& Laurence (1996) suggested two other neutral formulations, given by

$$
\phi_{i j}^{*}-\nabla \cdot\left(L^{2} \nabla \phi_{i j}^{*}\right)=\phi_{i j}^{h},
$$

and

$$
\phi_{i j}^{*}-L \nabla^{2}\left(L \phi_{i j}^{*}\right)=\phi_{i j}^{h} .
$$

These new formulations have been derived empirically and suffer form a lack of justifications, as emphasized by the authors themselves. This work aims, through a DNS analysis, to provide a more solid basis for deriving such modifications to the model. The central idea is that the correlation function $f\left(\boldsymbol{x}, \boldsymbol{x}^{\prime}\right)$ cannot be represented by a simple exponential function, contrary to what was assumed by Durbin (1991). Indeed, the presence of the wall induces a blocking effect, leading to not only an elongation of the turbulent structures, but also an asymmetry in the direction normal to the wall. Fluctuating quantities are correlated over a shorter distance in the direction toward the wall than away from it. There is plenty of experimental evidence (Hanjalić \& Launder 1972; Sabot 1976) of this feature in two-point correlations between components of the fluctuating velocity, and one can reasonably deduce that the two-point correlations between the fluctuating velocity and the Laplacian of the pressure gradient behave in a similar manner. The use of the symmetrical correlation function (2.7) leads to overweighting the region between the point and the wall, which may be the reason for the spurious behaviour of the elliptic relaxation equation in the logarithmic layer. This issue will be explored in the present DNS analysis, in order to understand how modifications to the elliptic relaxation model, such as those proposed by Wizman et al. (1996), Laurence \& Durbin (1994) and Durbin \& Laurence (1996), can be derived.

\subsection{Channel flow database and post-processing}

The correlation function $f\left(\boldsymbol{x}, \boldsymbol{x}^{\prime}\right)$ involves the Laplacian of the pressure gradient, which contains three spatial derivatives. Therefore, a very accurate DNS database is needed. The channel flow simulation at $R e_{\tau}=590$ performed by Moser et al. (1999) was chosen because of its numerical accuracy, the large number of available statistical samples, and the relatively high Reynolds number. This flow was computed on a grid of $384 \times 257 \times 384$ points in streamwise $(x)$, wall-normal $(y)$ and spanwise $(z)$ directions, respectively. The computational domain is $2 \pi h, 2 h$ and $\pi h$ in $x, y$ and $z$, where $h$ denotes the channel halfwidth. The simulation code is based on a spectral method for spatial derivatives (Fourier series in $x$ and $z$, and Chebychev polynomials in $y$ ), and a semi-implicit scheme for time integration. For statistical averaging, a total of 75 fields (restart files) are available, in addition to the spatial averaging in $x$ - and $z$-directions.

The two-point correlations between the fluctuating velocities and the Laplacian of the pressure gradient are needed for evaluating the correlation function $f\left(\boldsymbol{x}, \boldsymbol{x}^{\prime}\right)$. First, the 
Laplacian of the total pressure is evaluated from the velocity field,

$$
\nabla^{2} \widetilde{p}=-\rho \frac{\partial \widetilde{u}_{i}}{\partial x_{j}} \frac{\partial \widetilde{u}_{j}}{\partial x_{i}}
$$

where the tilde denotes total quantities, using the same Fourier/Chebychev spectral method as for the DNS. The gradient of this quantity is calculated using Fourier spectral derivatives in $x$ and $z$, and fourth order finite differences in $y$. The one-point and twopoint correlations between this gradient and the total velocity components are then calculated, and corresponding mean quantities are finally subtracted out in order to obtain correlations between fluctuating quantities. The post-processing computations are very time consuming, since they involve calculations of two-point correlations and averaging over 75 restart files and $x-z$ planes. As a practical matter, calculations are performed at 7 representative $y$-locations only, for separations restricted to $x-y, x-z$ and $y-z$ planes.

\section{Results and discussion}

\subsection{The wall echo}

The wall echo concept originates from the form of the integral equation of the redistribution term in a semi-infinite space bounded by an infinite plane $\left(\Omega=\mathbf{R} \times \mathbb{R}^{+} \times \mathbf{R}\right)$. In this domain, it can easily be shown that the Green function is

$$
G_{\mathbf{R} \times \mathbf{R}^{+} \times \mathbb{R}}\left(\boldsymbol{x}, \boldsymbol{x}^{\prime}\right)=-\frac{1}{4 \pi r}-\frac{1}{4 \pi r^{*}},
$$

where $r^{*}=\left\|\boldsymbol{x}^{*}-\boldsymbol{x}\right\|, \boldsymbol{x}^{\prime *}$ being the image of $\boldsymbol{x}^{\prime}$ in the plane $y=0$. The image term is due to the homogeneous Neumann boundary condition. Thus, at each point of the domain, the pressure fluctuation is the sum of the fluctuations generated by the velocity field and its reflection in the wall, which is called wall echo by analogy with acoustics. This echo is instantaneous, since the fluid is considered as incompressible.

Since the paper of Launder, Reece \& Rodi (1975), it has been widely accepted in the turbulence community that this wall echo is responsible for the reduction of the amplitude of the energy redistribution between components of the Reynolds stress. In second moment closures, wall echo terms are frequently incorporated to account for this phenomenon, as proposed by Gibson \& Launder (1978). These terms have proven to be effective for simple flows but are often not well defined in complex geometries.

The conclusion that wall echo reduces redistribution is, however, incorrect. Since the image term in the Green function appears with the same sign as the principal term, it actually induces an amplification of the redistribution.

In the case of a channel $(\Omega=\mathbf{R} \times[0,1] \times \mathbf{R})$, the Green function is easily derived after taking Fourier transform in homogeneous directions. However, working in the spectral space is not relevant in the present study. In order to examine the problem of the wall echo effect in a channel, an approximate Green function is needed.

It can be shown (cf. appendix A) that the simplest approximation to the Green function which is valid down to the wall and accommodates the boundary conditions is $G_{\Omega}\left(\boldsymbol{x}, \boldsymbol{x}^{\prime}\right) \approx H\left(\boldsymbol{x}, \boldsymbol{x}^{\prime}\right)$, with

$$
H\left(\boldsymbol{x}, \boldsymbol{x}_{0}{ }_{0}\right)=-\frac{1}{4 \pi\left\|\boldsymbol{x}_{-1}^{\prime}-\boldsymbol{x}\right\|}-\frac{1}{4 \pi\left\|\boldsymbol{x}_{0}-\boldsymbol{x}\right\|}-\frac{1}{4 \pi\left\|\boldsymbol{x}_{1}-\boldsymbol{x}\right\|},
$$

where $\boldsymbol{x}^{\prime}{ }_{-1}$ and $\boldsymbol{x}_{1}{ }_{1}$ are the images of $\boldsymbol{x}^{\prime}{ }_{0}$ in the walls located at $y=0$ and $y=1$, respectively. 

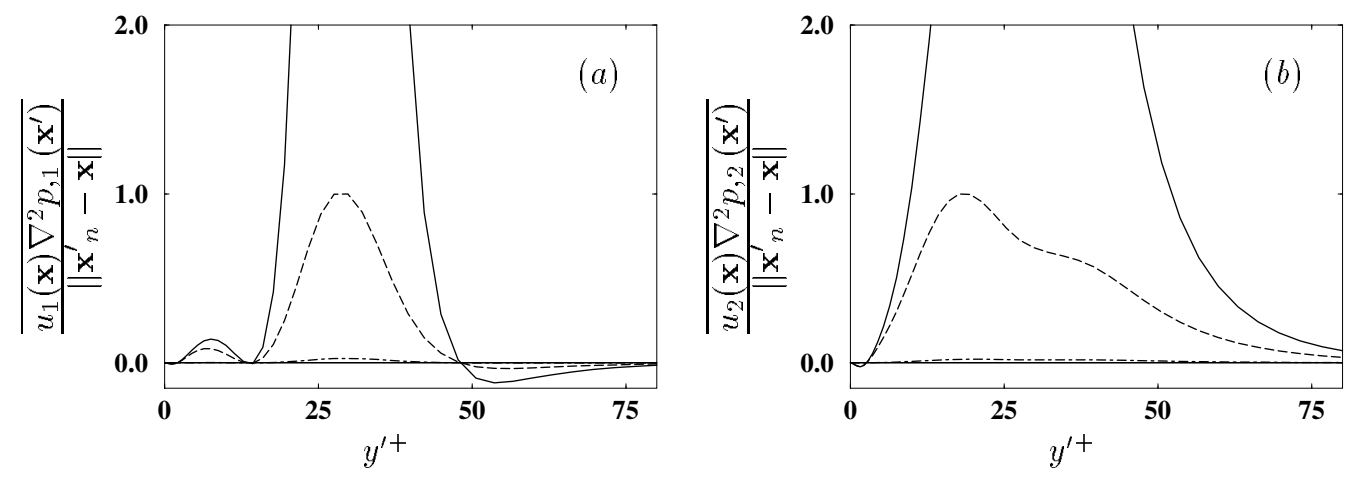

Figure 1. Comparison of the three terms in the integrand of (4.3) at $y^{+}=30$ for separations in $y$-direction. (a) Integrand of $\phi_{11}^{*} ;(b)$ Integrand of $\phi_{22}^{*} .-$ principal term $(n=0)$; - - image term due to the wall at $y^{+}=0(n=-1) ;---$ image term due to the wall at $y^{+}=1180$ $(n=1)$. The vertical coordinate is arbitrarily normalized such that the maximum of $n=-1$ term is 1 .

With this approximation, the redistribution term (2.4) can be written as

$$
\rho \phi_{i j}^{*}(\boldsymbol{x})=-\frac{1}{4 \pi} \int_{\Omega} \Psi_{i j}\left(\boldsymbol{x}, \boldsymbol{x}_{0}^{\prime}\right)\left(\frac{1}{\left\|\boldsymbol{x}_{-1}^{\prime}-\boldsymbol{x}\right\|}+\frac{1}{\left\|\boldsymbol{x}_{0}^{\prime}-\boldsymbol{x}\right\|}+\frac{1}{\left\|\boldsymbol{x}_{1}^{\prime}-\boldsymbol{x}\right\|}\right) \mathrm{d} V\left(\boldsymbol{x}_{0}^{\prime}\right) .
$$

The three terms in the integrand, calculated from the DNS database, are shown in figure 1, for the components $\phi_{11}^{*}$ and $\phi_{22}^{*}$. The solid line, representing the principal term, has been truncated because it goes to infinity at $y^{\prime}-y=0$. It can be seen that the image term arising from the far wall at $y^{+}=1180$ is negligible, but not the term due to the near wall at $y^{+}=0$. The exact weight of each term has not been evaluated, since it involves integral of two-point correlations over separations in all directions, which have not been calculated. Nevertheless, following Bradshaw (1973), the amplitude of each term can be roughly estimated: the two-point correlation can be approximated by a constant inside a sphere of radius $L$, and zero outside of it. If $L / y$ and $y / 2 h$ are both small, (4.3) yields

$$
\rho \phi_{i j}^{*}(\boldsymbol{x})=-\frac{L^{2}}{2} \Psi_{i j}(\boldsymbol{x}, \boldsymbol{x})\left(\frac{L}{3 y}+1+\frac{L}{6 h}\right) .
$$

This result shows that, if the length scale is $L=\kappa y$, the ratio of the image term $(n=-1)$ to the principal term is approximately $14 \%$. For the second image term $(n=1)$, the ratio is $0.07 y / h$, which is approximately $0.4 \%$ at $y^{+}=30$. Thus, the first image term cannot be neglected, as long as the length scale is of the order of $\kappa y$. Note that, contrary to Bradshaw (1973), who concluded that the presence of the wall changes the redistribution term by \pm 0.14 , we can affirm that the sign is positive, i.e., the wall echo actually increases the redistribution. Indeed, it can be seen in figure 1 that the contribution of the image terms to the integral is of the same sign as that of the principal term. The weight of the negative excursions of the first image term is far too small to change the sign of the total contribution of this term.

Thus, an interesting and important conclusion can be drawn. The actual wall echo leads to an amplification of the redistribution, contrary to the common belief. The Gibson \& Launder (1978) type terms, which have been found useful in reproducing the strong anisotropy near walls, have the correct damping effect, but for the wrong reason. This damping cannot be traced to the wall echo effect, i.e., the appearance of an image term in the Green function. Rather, it is caused by the damping of the two-point correlation 
itself, due to the modification of the turbulence by the wall, as a consequence of the nonlocal nature of the redistribution term in the strongly inhomogeneous boundary layer. The success of Gibson \& Launder (1978) type terms lies in the introduction of non-local effects through the explicit reference to the wall-distance, and we believe the more recent attempts to replace these terms by highly nonlinear models is not the best route to follow. Non-local models, such as the elliptic relaxation model, appears more suitable for accounting for the influence of the wall on the turbulence.

\subsection{Asymmetry in y-direction}

In this section the shape of the correlation function defined by (2.6) is evaluated. One problem that arises is that this function should depend on the component $(i, j)$. Indeed, a different correlation function can be evaluated for each component of $\phi_{i j}^{*}$ by

$$
f\left(\boldsymbol{x}, \boldsymbol{x}^{\prime}\right)=\frac{\overline{u_{\alpha}(\boldsymbol{x}) \nabla^{2} \frac{\partial p}{\partial x_{\beta}}\left(\boldsymbol{x}^{\prime}\right)}+\overline{\overline{u_{\beta}(\boldsymbol{x}) \nabla^{2} \frac{\partial p}{\partial x_{\alpha}}\left(\boldsymbol{x}^{\prime}\right)}}}{\overline{u_{\alpha}\left(\boldsymbol{x}^{\prime}\right) \nabla^{2} \frac{\partial p}{\partial x_{\beta}}\left(\boldsymbol{x}^{\prime}\right)}+\overline{u_{\beta}\left(\boldsymbol{x}^{\prime}\right) \nabla^{2} \frac{\partial p}{\partial x_{\alpha}}\left(\boldsymbol{x}^{\prime}\right)}},
$$

without summation over Greek indices. Thus, it is impossible to derive a model for $f$ which matches all the DNS results.

In fact, the most general relation between two-point correlation and one-point correlation tensors, if they are assumed to be linearly connected, is

$$
\Psi_{i j}\left(\boldsymbol{x}, \boldsymbol{x}^{\prime}\right)=f_{i j k l}\left(\boldsymbol{x}, \boldsymbol{x}^{\prime}\right) \Psi_{k l}\left(\boldsymbol{x}^{\prime}, \boldsymbol{x}^{\prime}\right) .
$$

In this relation, the fourth order tensor $f_{i j k l}$ involves 81 coefficients, but their number can be dramatically reduced by using symmetry properties, as shown by Naot, Shavit \& Wolfshtein (1973). However, this type of relation remains too complicated for the purpose of accounting for the non-local effect. Therefore, a simple scalar correlation function is used, keeping in mind that it can only represent approximately the non-local effect. The following results must be interpreted in this sense.

The correlation functions evaluated from (4.5) are shown in figure 2. The correlation functions in figures $2(a)$ and $2(b)$ correspond respectively to $\phi_{11}^{*}$ and $\phi_{22}^{*}$. Some features which can be seen in this figures are rather favourable to the elliptic relaxation model: first, the correlation functions exhibit sharp peaks, which is consistent with the use of an exponential function to model it; secondly, the correlation lengths for both components 11 and 22 evolve in a similar way when the fixed location moves away from the wall.

However, some other features do not agree with the simple model assumptions. First, the peaks in figure $2(a)$ are much broader than these in figure $2(b)$, indicating that the correlation length scale is not the same for all the components. Secondly, the correlation function exhibits negative excursions, at locations close to the wall for the 11 component, and everywhere in the channel for the 22 component. These features, which are very similar to those observed in the two-point velocity correlations in boundary layer experiments (Grant 1958; Favre, Gaviglio \& Dumas 1957; 1958), call into question the modelling of the correlation function by a simple exponential function. Nevertheless, as emphasized previously, the model does not intend to represent exactly the two-point correlations, but only to globally account for the non-local effect. Moreover, it will be shown in the following sections that the correlation length scale, evaluated as the half-width of the peak of the correlation function, is very similar to the integral scale. This indicates that the negative excursions do not contribute significantly to the integral, and thus do not strongly influence the redistribution term.

The main feature which arises from these figures is that the correlation functions do 

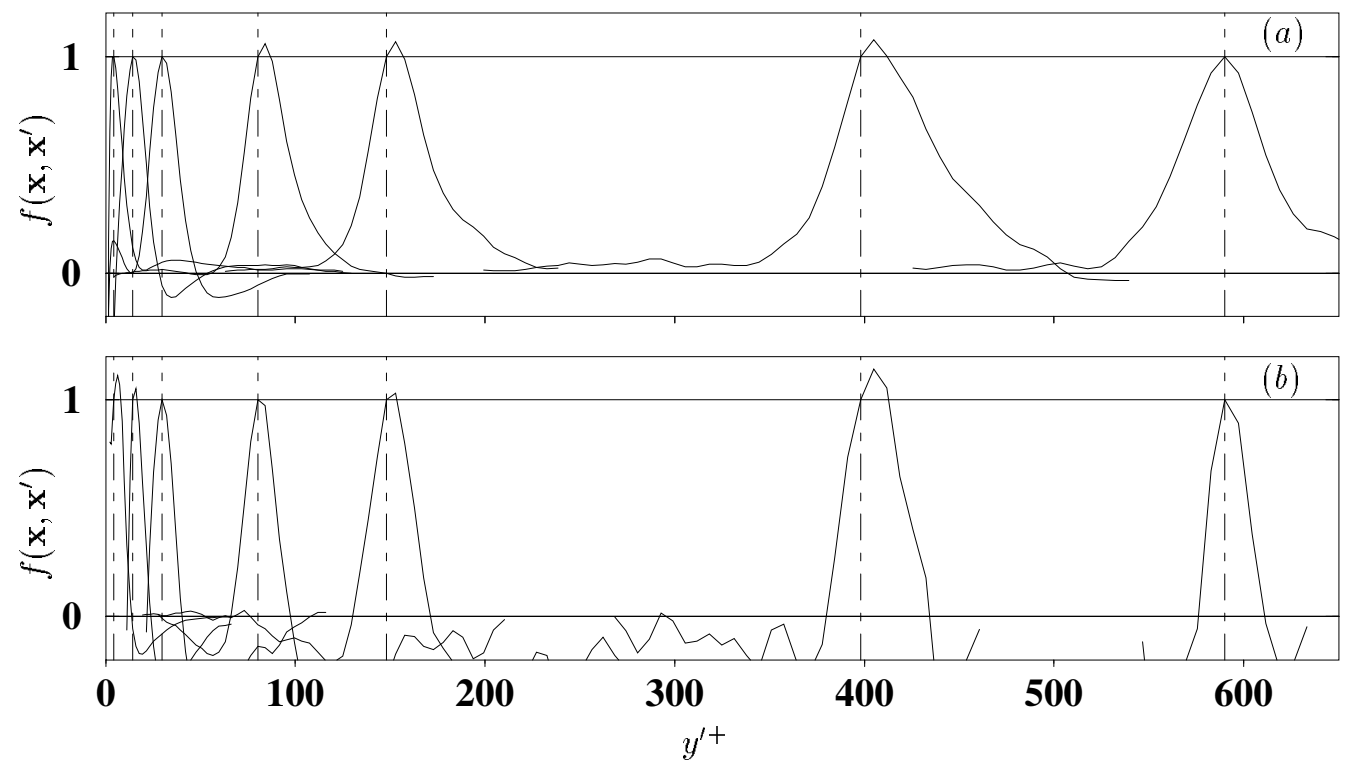

FIGURE 2. Shape of the correlation function calculated from the DNS database at 7 different $y$-locations: $y^{+}=4 ; y^{+}=14 ; y^{+}=30 ; y^{+}=80 ; y^{+}=150 ; y^{+}=400 ; y^{+}=590$. Separations in the $x$ - and $z$-directions are zero. $f\left(\boldsymbol{x}, \boldsymbol{x}^{\prime}\right)$ is evaluated from (4.5) with: $(a) \alpha=\beta=1 ;(b)$ $\alpha=\beta=2$. For clarity, curves have been truncated at large separations.

not have symmetrical shapes. It can also be seen that the maxima of the peaks do not generally occur at zero separation. As it is defined, the correlation function is not restricted to be smaller than one. Values greater than one can occur if the amplitude (rms value) of the fluctuating velocity varies rapidly with distance to the wall.

The main implication of this asymmetrical shape is that the points $\boldsymbol{x}^{\prime}$ located between the fixed point $\boldsymbol{x}$ and the wall contribute less to the integral than points $\boldsymbol{x}^{\prime}$ toward the core of the flow. Hence, modelling the correlation function by a symmetrical exponential function leads to overweighting the points toward the wall, thus increasing the value of the integral (a formal proof of this can be found in Appendix B). This problem is illustrated by figure 3 , for the 22 component. The correlation functions calculated from the DNS using (4.5), as well as from two models, are shown in figure $3(a)$. The first one is the original model, i.e., a simple exponential function, whereas the second takes into account the gradient of the length scale in order to reproduce the previously noted asymmetry. In figure $3(b)$, two-point correlations obtained by multiplying the one-point correlation from the DNS by the modelled correlation function are shown. It can be seen that, with the original model, points toward the wall are overweighted, whereas with the corrected model, the two-point correlations are much better reproduced in this region.

The overweighting of the two-point correlation for separations in the direction of the wall is thought to be at the origin of the erroneous amplification of the redistribution in the logarithmic layer noted in $\S 3.1$. As shown in figure $3(b)$, introducing a dependence on the gradient of the length scale in the model to account for the asymmetry of the correlation function corrects this deficiency. It will be shown, in $\S 5.2$, that a new formulation of the elliptic relaxation function can be derived from this extended model and, in $\S 5.3$, that this formulation overcomes the shortcomings of the original one. 


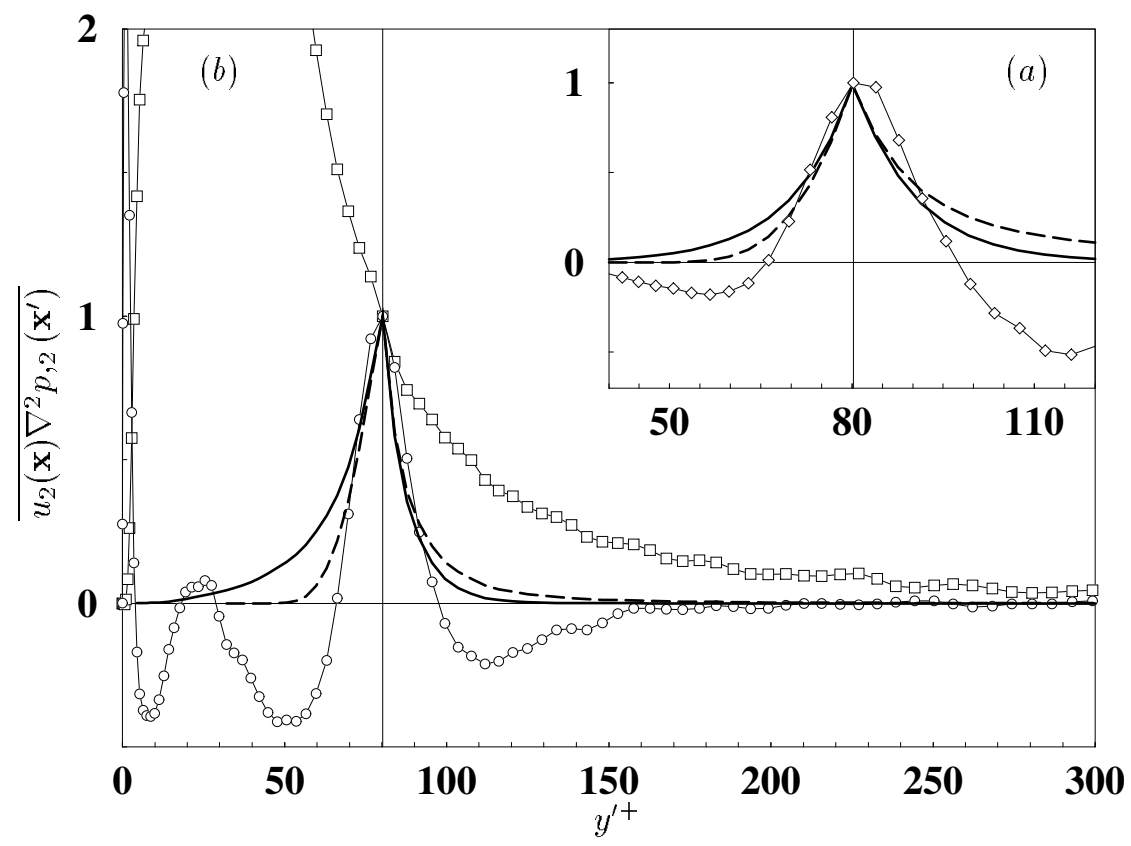

Figure 3. Two-point correlation $\Psi_{22}^{\mathrm{DNS}}\left(\boldsymbol{x}, \boldsymbol{x}^{\prime}\right)$ at $y^{+}=80$ obtained using two different correlation functions. (a) Correlation function: $\diamond f^{\mathrm{DNS}}\left(\boldsymbol{x}, \boldsymbol{x}^{\prime}\right)$; - original model $f_{1}\left(\boldsymbol{x}, \boldsymbol{x}^{\prime}\right)=\exp (-r / L)$; - - asymmetrical model $f_{2}\left(\boldsymbol{x}, \boldsymbol{x}^{\prime}\right)=\exp \left(-r /\left(L+\left(\boldsymbol{x}^{\prime}-\boldsymbol{x}\right) \cdot \boldsymbol{\nabla} L\right)\right)$. (b) $\square$ One-point correlation $\Psi_{22}^{\mathrm{DNS}}\left(\boldsymbol{x}^{\prime}, \boldsymbol{x}^{\prime}\right)$; O Two-point correlation $\Psi_{22}^{\mathrm{DNS}}\left(\boldsymbol{x}, \boldsymbol{x}^{\prime}\right)\left(=\Psi_{22}^{\mathrm{DNS}}\left(\boldsymbol{x}^{\prime}, \boldsymbol{x}^{\prime}\right) f^{\mathrm{DNS}}\left(\boldsymbol{x}, \boldsymbol{x}^{\prime}\right)\right)$;

Two-point correlation given by the original model $\Psi_{22}\left(\boldsymbol{x}, \boldsymbol{x}^{\prime}\right)=\Psi_{22}^{\mathrm{DNS}}\left(\boldsymbol{x}^{\prime}, \boldsymbol{x}^{\prime}\right) f_{1}\left(\boldsymbol{x}, \boldsymbol{x}^{\prime}\right)$;

Two-point correlation given by the asymmetrical model $\Psi_{22}\left(\boldsymbol{x}, \boldsymbol{x}^{\prime}\right)=\Psi_{22}^{\mathrm{DNS}}\left(\boldsymbol{x}^{\prime}, \boldsymbol{x}^{\prime}\right) f_{2}\left(\boldsymbol{x}, \boldsymbol{x}^{\prime}\right)$.

\subsection{Anisotropy}

The asymmetry in the direction normal to the wall, which has been emphasized in the previous section, is not the only anisotropy. The purpose of this section is to point out that the correlation function does not possess the same shape for separations in the three principal directions. This anisotropy is visible in figures 4 and 5, for correlation functions corresponding to $\phi_{11}^{*}$ and $\phi_{22}^{*}$, respectively. At locations very close to the wall (figures $4 a, b$ and $5 a, b$ ), the correlation function is strongly elongated in the streamwise direction, in particular for the 22-component (figure $5 a, b$ ). This is mainly due to the fact that, in the $y$-direction, the correlation length scale is constrained by the presence of the wall (wall-blocking effect). In figures 4 and 5 , it can be noted that $f$ goes to infinity at the wall. For instance, for the 22-component, $f$ behaves as $y^{\prime-2}$ when $\boldsymbol{x}^{\prime}$ approaches the wall. This is a consequence of its definition (4.5): the two-point correlation only contains $u_{2}(y)$, which is constant with respect to $y^{\prime}$, whereas the one-point correlation involves $u_{2}\left(y^{\prime}\right)$, which behaves as $y^{\prime 2}$.

Away from the wall, the correlation function becomes increasingly isotropic (figures $4 c^{-}$ $f$ and $5 c-f)$. In the center of the channel, it is completely isotropic for the 11-component (figure $4 g$ ), but still slightly elongated in the streamwise direction for the 22-component (figure $5 g$ ). These features are also conspicuous in figures $6-11$. In the figures $(a),(b)$ and $(c)$, the shapes of the correlation functions are shown for separations in $x-y, x-z$ and $y-z$ planes, respectively. The iso-correlation contours in these planes are shown in the figures $(d)$. The elongation of the correlation function, which is consistent with the well-known elongation of turbulent structures near the wall, clearly appears in figures 6 


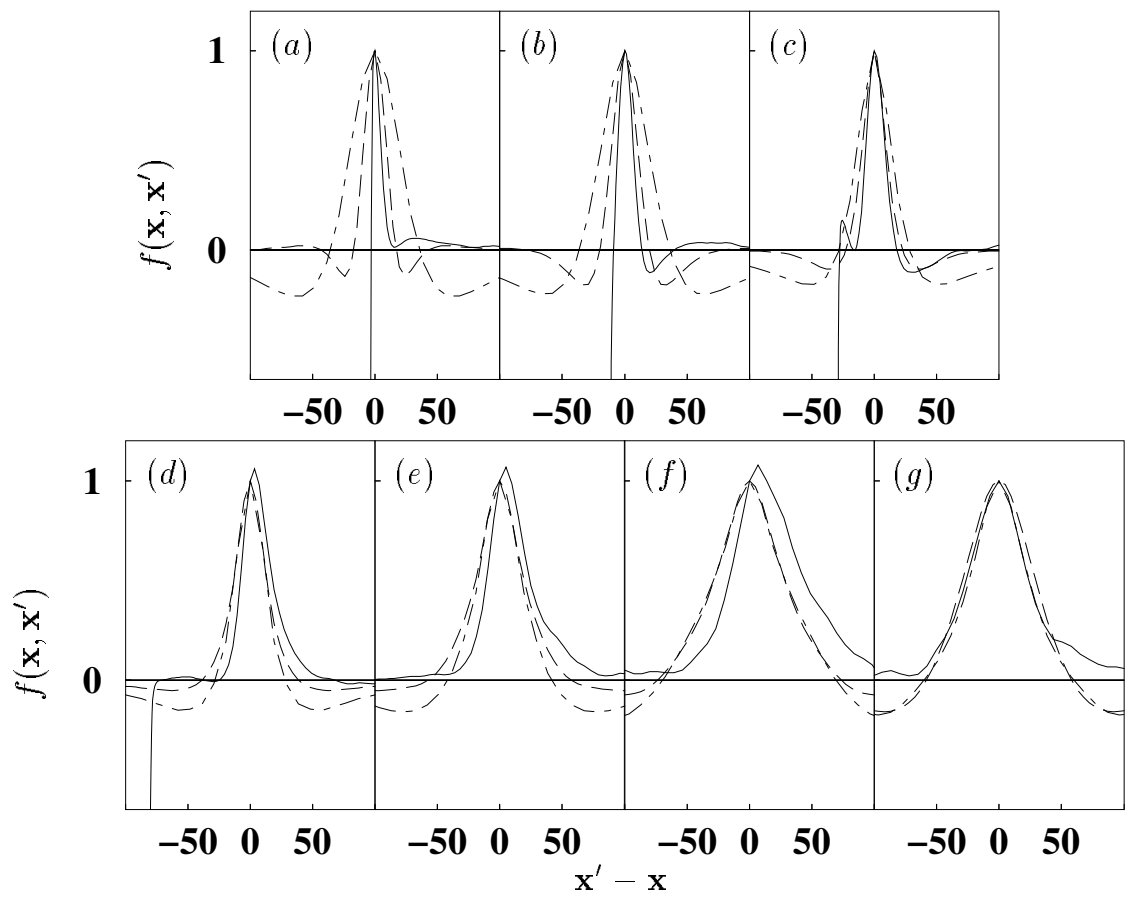

Figure 4. Shape of the correlation function defined by (4.5) with $\alpha=\beta=1$, evaluated from the DNS data, in the 3 principal directions, at 7 different $y$-locations. $(a) y^{+}=4 ;(b) y^{+}=14 ;(c)$ $y^{+}=30 ;(d) y^{+}=80 ;(e) y^{+}=150 ;(f) y^{+}=400 ;(g) y^{+}=590$. Separations: $--x$-direction $(\Delta y=\Delta z=0) ;-y$-direction $(\Delta x=\Delta z=0) ;---z$-direction $(\Delta x=\Delta y=0)$.
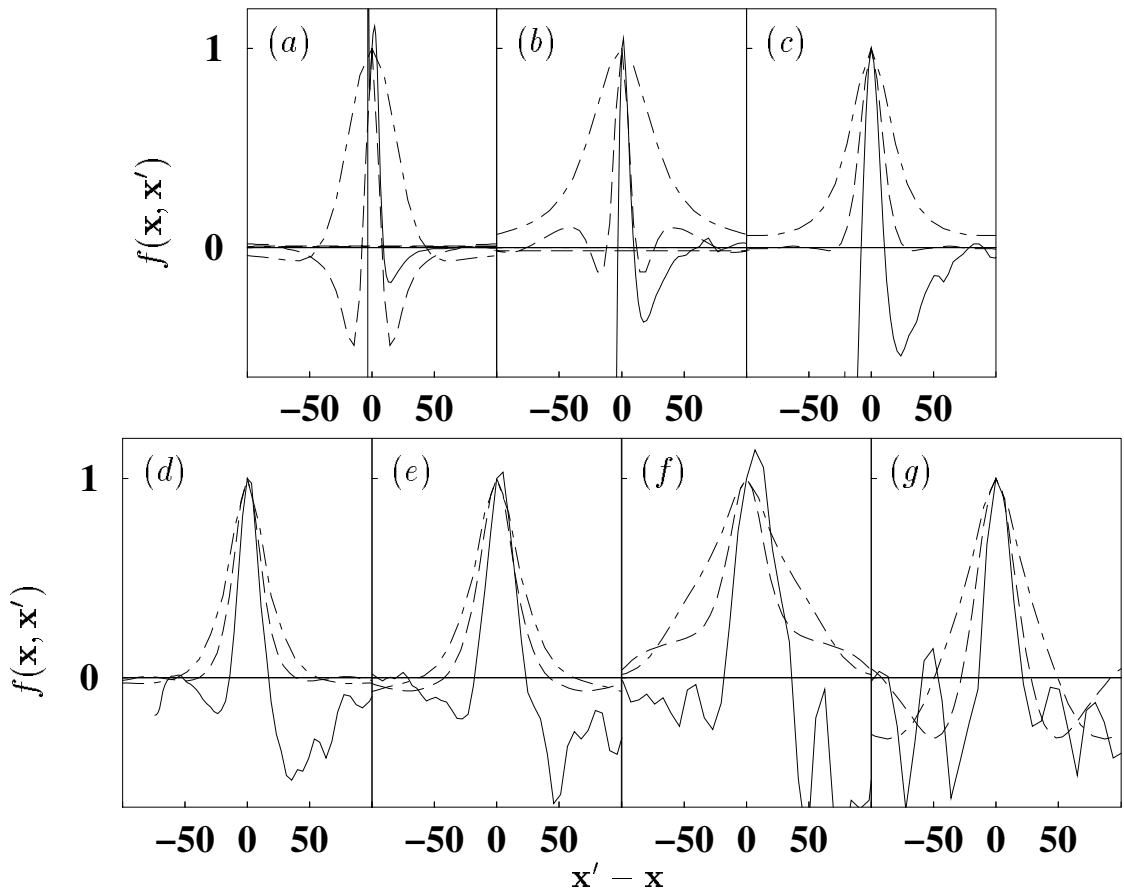

Figure 5. Same as figure 4 for $\alpha=\beta=2$. 


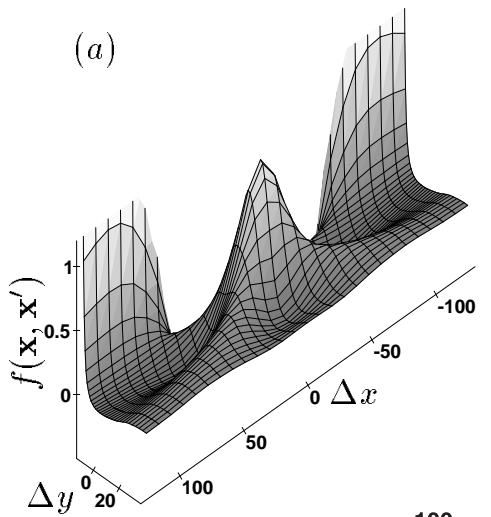

(b)

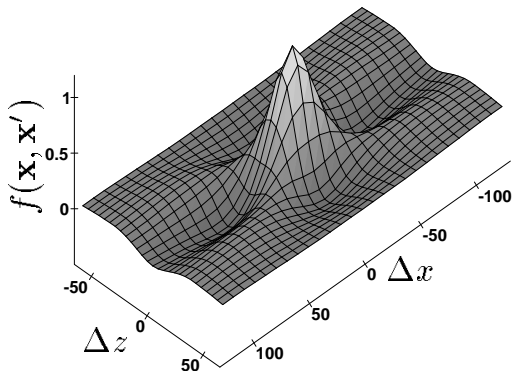

(c)

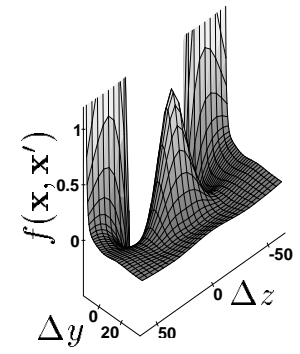

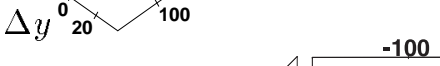

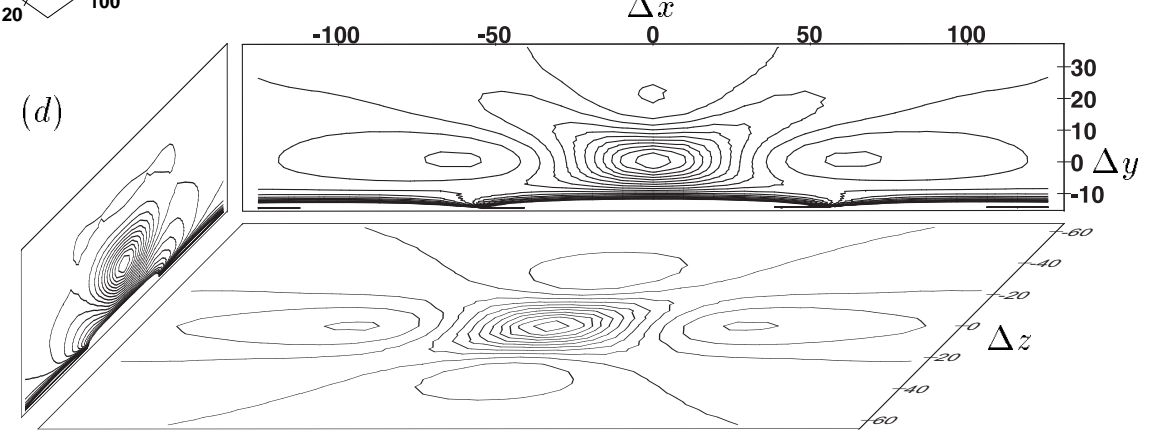

Figure 6. Shape of the correlation function defined by (4.5) with $\alpha=\beta=1$ at $y^{+}=14$. (a) Separation in $x-y$ plane; $(b)$ Separation in $x-z$ plane; $(c)$ Separation in $y-z$ plane; $(d)$ Iso-correlation contours in these three planes ( 21 contours from 1 to -1 are plotted).

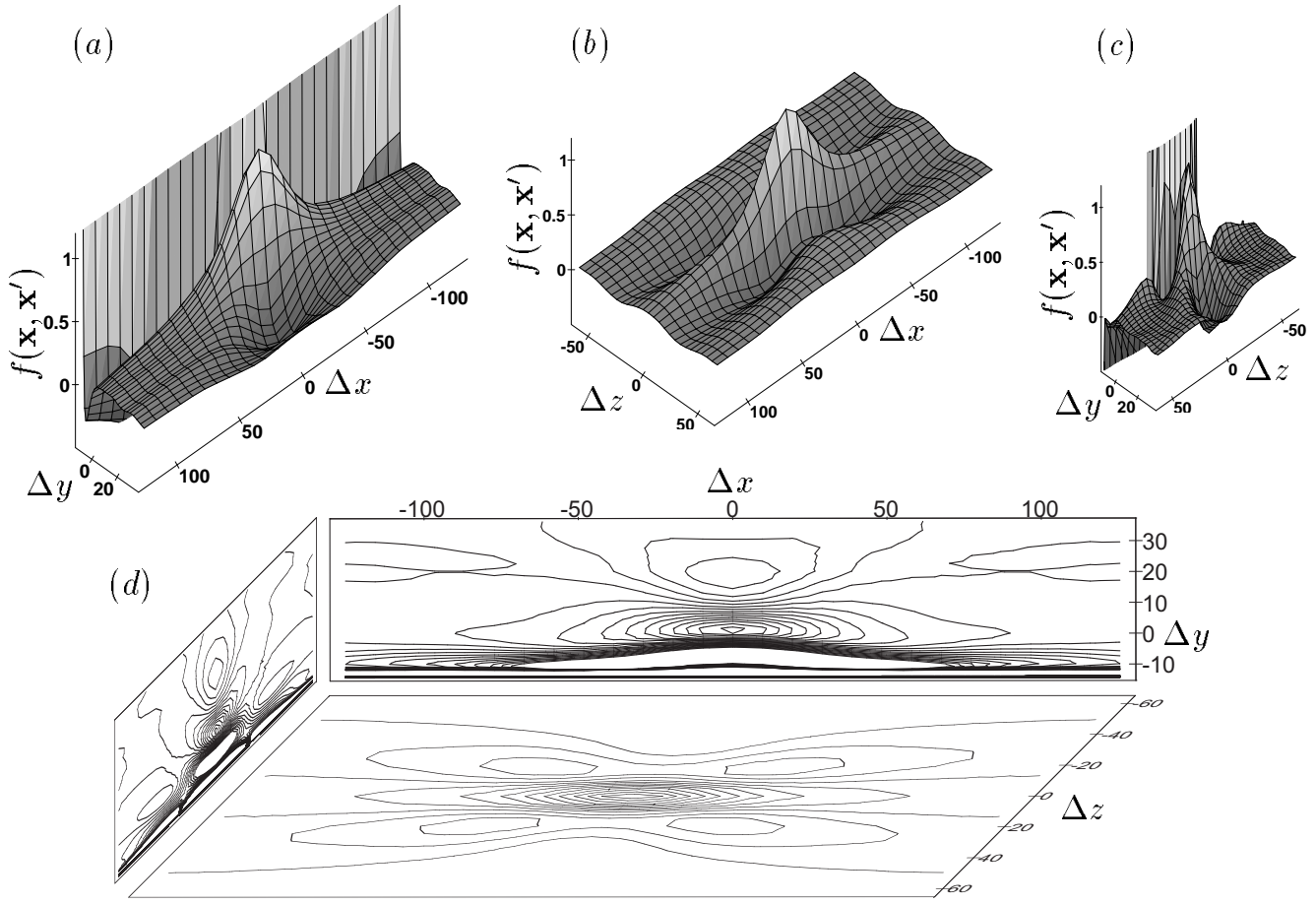

Figure 7 . Same as figure 6 with $\alpha=\beta=2$ at $y^{+}=14$. 


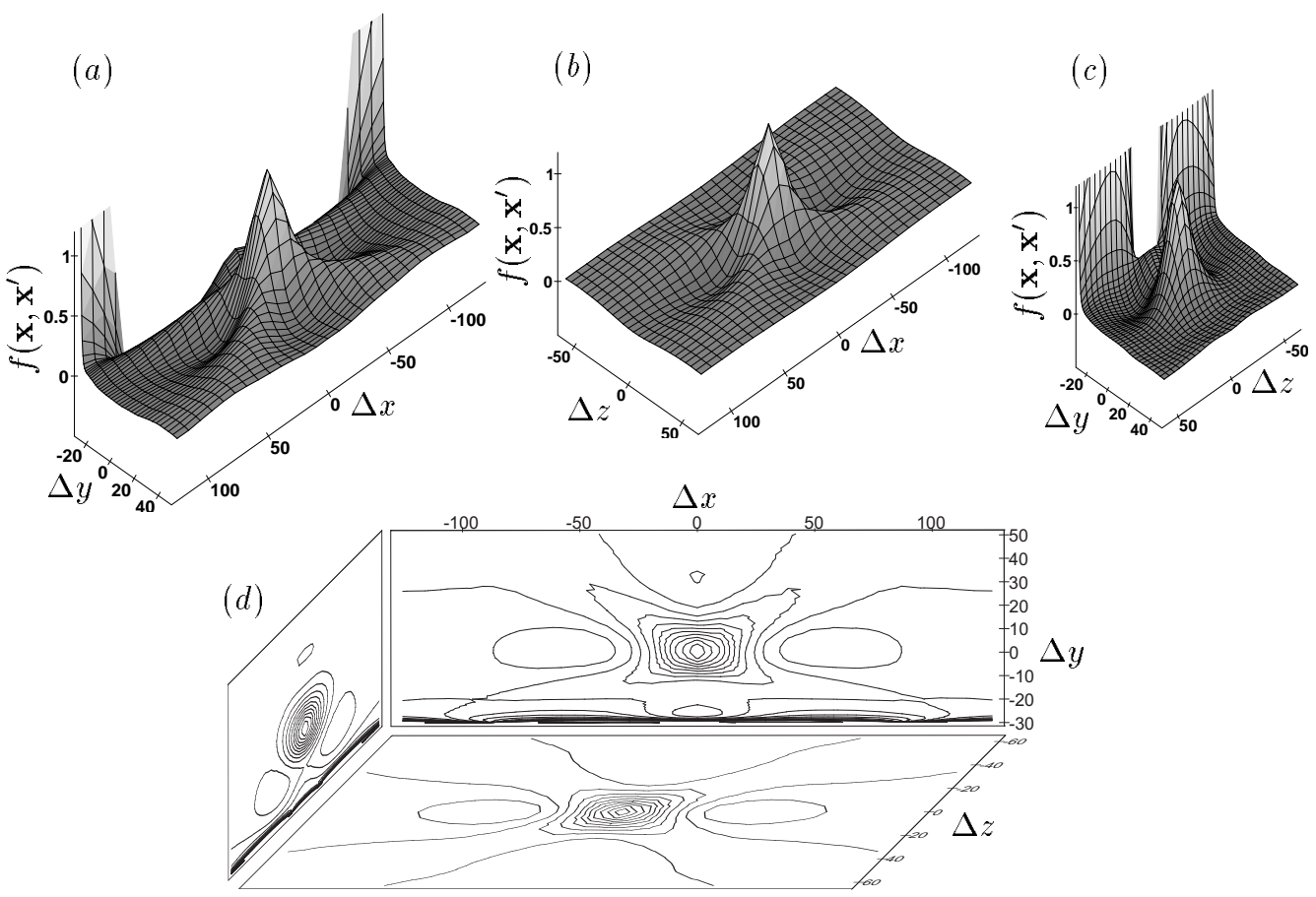

Figure 8 . Same as figure 6 with $\alpha=\beta=1$ at $y^{+}=30$.
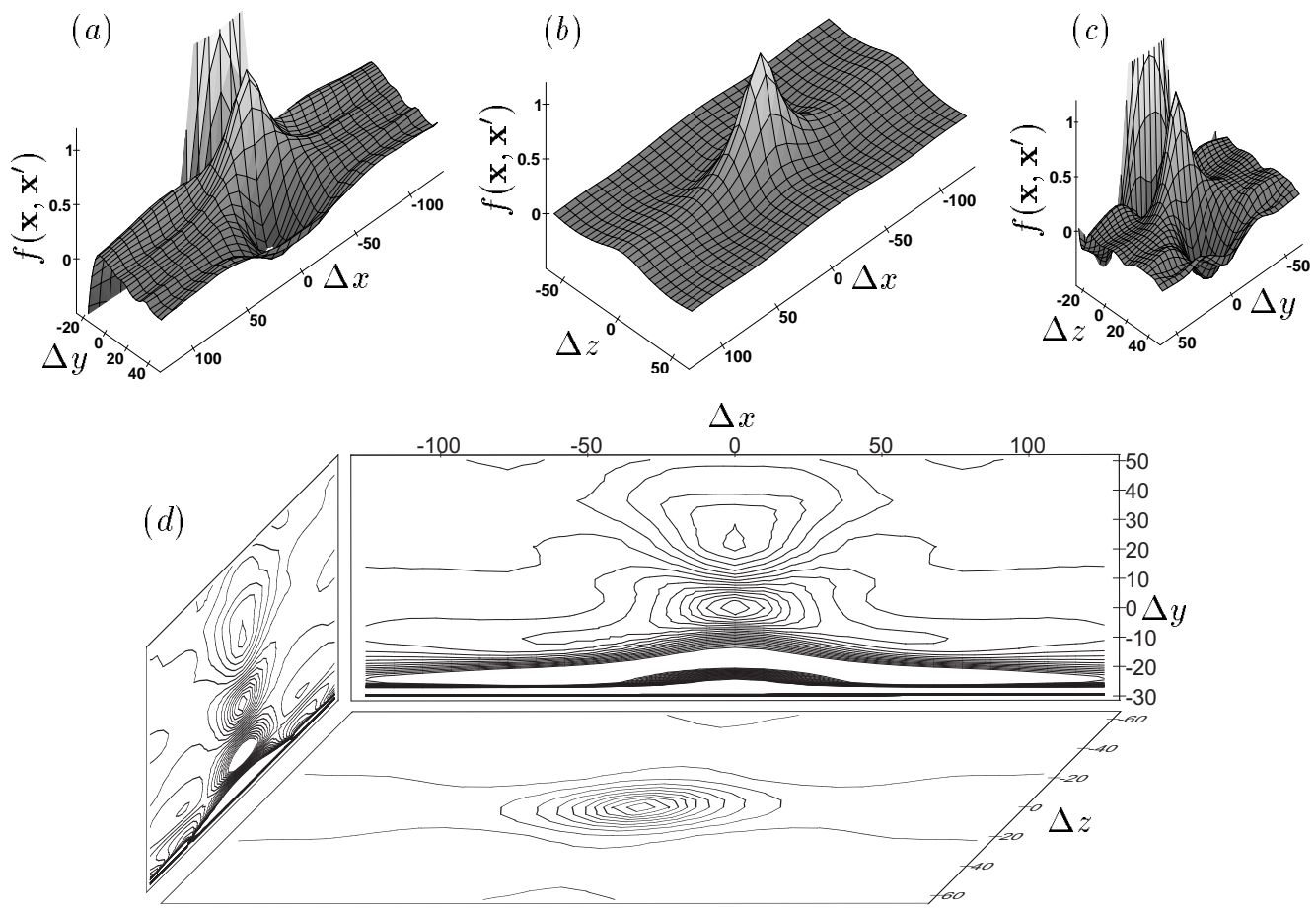

Figure 9 . Same as figure 6 with $\alpha=\beta=2$ at $y^{+}=30$. 

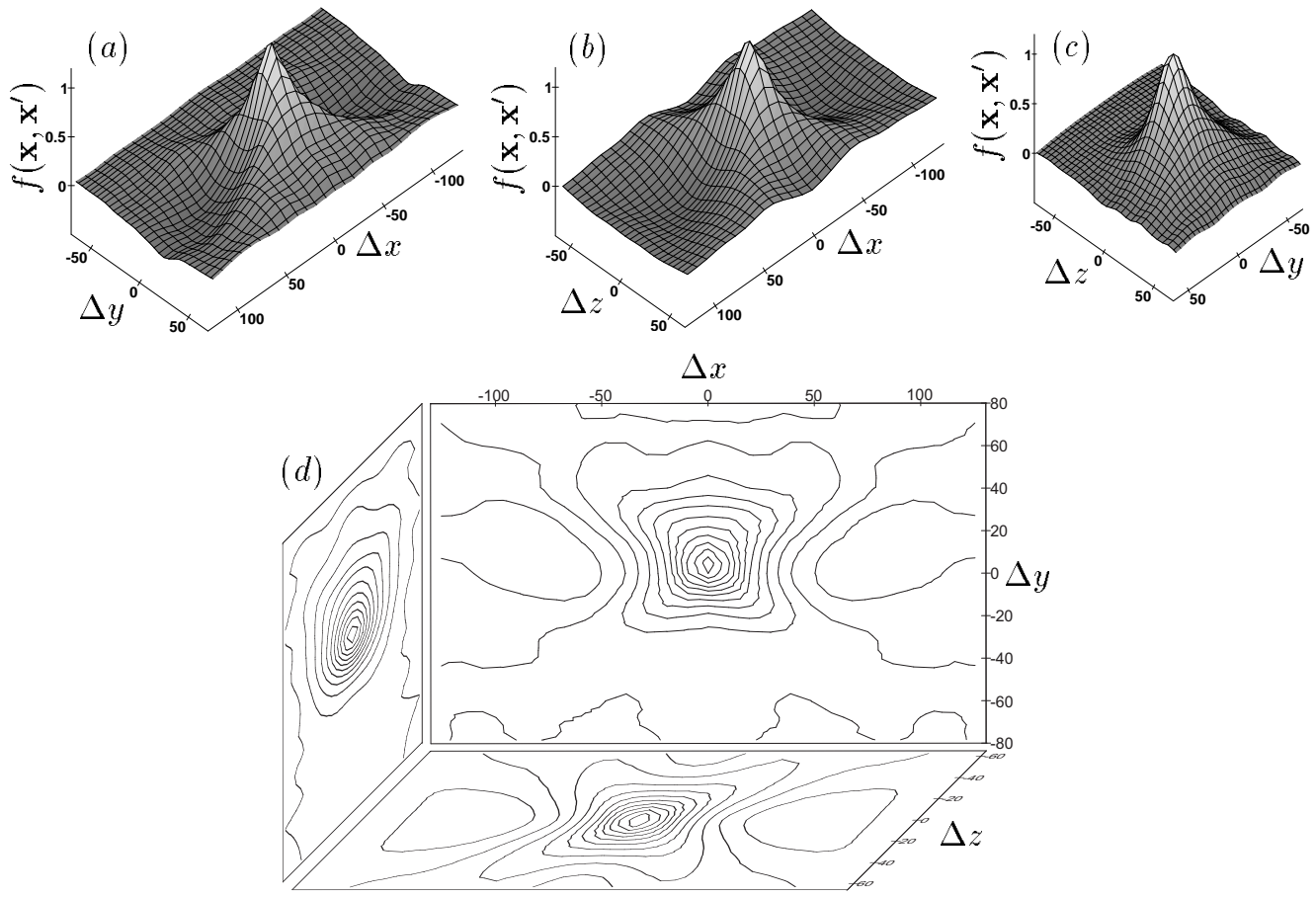

Figure 10. Same as figure 6 with $\alpha=\beta=1$ at $y^{+}=150$.
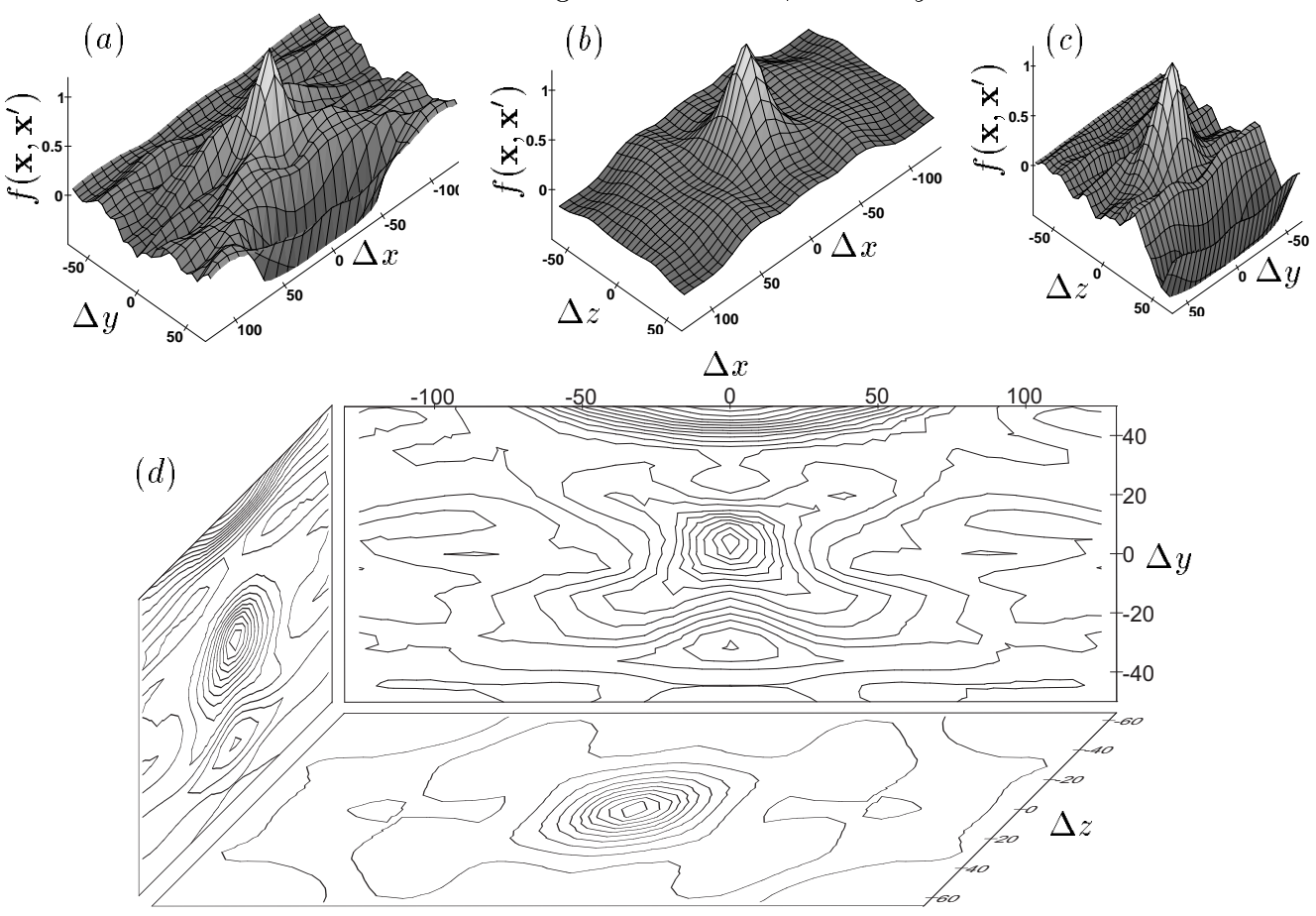

Figure 11. Same as figure 6 with $\alpha=\beta=2$ at $y^{+}=150$. 

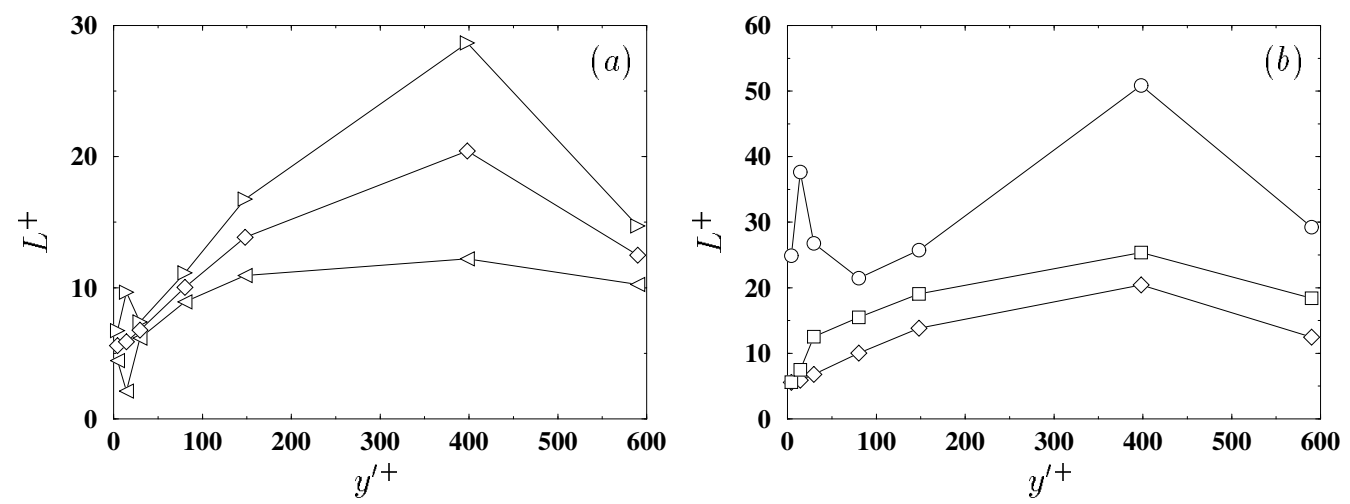

FIGURE 12. Length scales characterizing the correlation function corresponding to $\phi_{22}^{*}$ evaluated from the curves in figure 5. (a) Comparison of the length scales in $y$-direction: $\triangleleft$ Left length scale $L_{-e_{2}} ; \triangleright$ Right length scale $L_{e_{2}} ; \diamond$ Central length scale $L=\frac{1}{2}\left(L_{-e_{2}}+L_{e_{2}}\right)$. (b) Comparison of the central length scales in the principal directions: $\bigcirc x$-direction; $\diamond y$-direction; $\square z$-direction.

and 7 , in particular for the 22-component. It gradually decreases when the fixed point $\boldsymbol{x}$ moves away from the wall (figures 8 and 9). Further away from the wall (figures 10 and 11), the elongation in streamwise direction is almost totally removed, but the asymmetry in $y$-direction, emphasized in $\S 4.2$, is visible.

The model does not account for the elongation in streamwise direction, since it uses a function of $r$, which does not distinguish different directions. This shortcoming cannot be responsible for the spurious amplification of the redistribution in the logarithmic layer, pointed out in $\S 3.1$, since in the case of a channel flow, the non-local effect does not act in the homogeneous directions $x$ and $z$. Nevertheless, in more complex flows, this feature of the correlation function can become significant. In $\S 5.1$, a new formulation of the elliptic relaxation equation will be proposed, allowing the introduction of different length scales in each directions, thus taking into account the anisotropy of the turbulent structures.

\subsection{Length scales}

It is noted that the function $\exp (-r / L)$ takes the value $1 / \mathrm{e}$ for $r=L$. Hence, the correlation length scale can be defined by the separation at which the correlation function takes this value. Notwithstanding its simplicity, this method provides an evaluation of a length scale $L_{\boldsymbol{m}}$, defined implicitly by

$$
f\left(\boldsymbol{x}, \boldsymbol{x}+L_{\boldsymbol{m}} \boldsymbol{m}\right)=\frac{1}{\mathrm{e}}
$$

in each direction $\boldsymbol{m}$, including the inhomogeneous direction. The drawback is that this definition only enables the characterization of the shape of the function at moderate separations and cannot account for more complex features, such as negative excursions.

Figure 12 shows the different length scales evaluated with this method from the correlation functions corresponding to $\phi_{22}^{*}$, depicted in figure 5 . In figure $12(a)$, three length scales in the $y$-direction are compared: the left length scale, defined by (4.7) with $\boldsymbol{m}=-\boldsymbol{e}_{2}\left(\boldsymbol{e}_{1}, \boldsymbol{e}_{2}\right.$ and $\boldsymbol{e}_{3}$ being the basis vectors in streamwise, wall-normal and spanwise directions, respectively); the right length scale, defined by (4.7) with $\boldsymbol{m}=\boldsymbol{e}_{2}$, and the central length scale, which is the algebraic mean of the two previous ones, i.e., the half-width of the correlation function. It can be noted that the asymmetry, already emphasized in $\S 4.2$, is rather strong in the main part of the flow. Except for the peculiar 


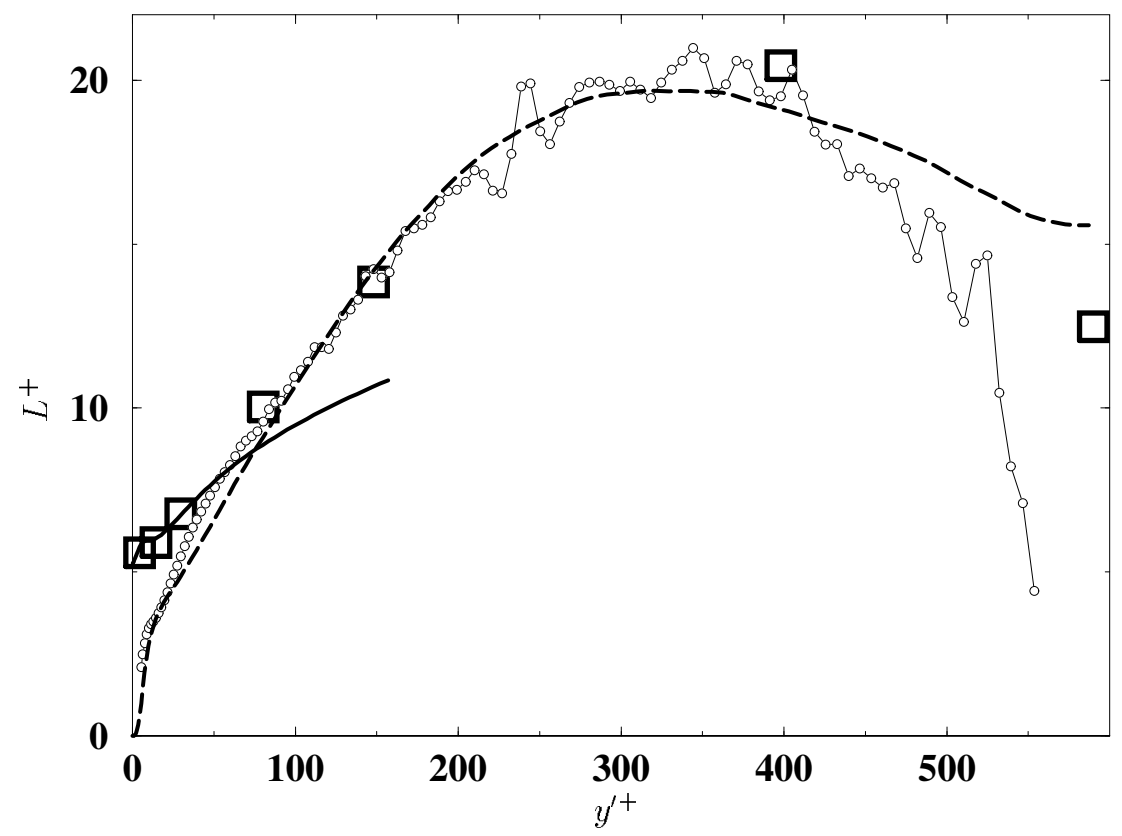

Figure 13. Comparison of the different length scale definitions for the $\phi_{22}^{*}$ component, evaluated from the DNS data: $\square$ Correlation length scale $L$ (central length scale in figure $12 a$ ), defined as the half-width of the correlation function; ○- o Integral length scale $L_{\text {int }}$ defined by (4.10) with $i=j=2$; - - Turbulent length scale used in the model $L_{T}=C_{L} k^{3 / 2} \varepsilon^{-1}\left(C_{L}=0.045\right)$; Kolmogorov length scale used near the wall in the model $L_{K}=C_{\eta} C_{L} \nu^{3 / 4} \varepsilon^{-1 / 4}\left(C_{\eta}=80\right)$.

behaviour at $y^{+}=14$, the asymmetry increases continuously with distance from the wall until it reaches a maximum, and then decreases in the vicinity of the centre. Note that the correlation function should be symmetrical at the centre if the data were perfectly statistically converged (the data have not been artificially symmetrized).

In figure $12(b)$, the central length scale in the $y$-direction, already plotted in figure $12(a)$, is compared to the length scales in the $x$ - and $z$-directions. This comparison provides a quantitative evaluation of the anisotropy of the correlation function, seen in figures $4-11$, and shows that the anisotropy of $\phi_{22}^{*}$ is very important everywhere in the domain. The three length scales show qualitatively the same variation across the channel, except below $y^{+}=50$, where the streamwise length scale exhibits a sharp spike, corresponding to the strong elongation of the iso-correlation contours emphasized in $\S 4.3$.

Figure 13 shows a comparison between the correlation length scale, defined as the half-width of the correlation function (the central length scale in figures $12 a, b$ ), and the integral length scale $L_{i n t}$. In order to define the latter, let us introduce the standard correlation function $\mathcal{F}$ (cf., for instance, Monin \& Yaglom 1975) to be used in the integral equation (2.4):

$$
\Psi_{i j}\left(\boldsymbol{x}, \boldsymbol{x}^{\prime}\right)=\Psi_{i j}(\boldsymbol{x}, \boldsymbol{x}) \mathcal{F}\left(\boldsymbol{x}, \boldsymbol{x}^{\prime}\right),
$$

where, contrary to (2.6), the one-point correlation is expressed in $\boldsymbol{x}$. The one-point correlation can then be taken outside the integral, which gives

$$
\rho \phi_{i j}^{*}(\boldsymbol{x})=\Psi_{i j}(\boldsymbol{x}, \boldsymbol{x}) \int_{\Omega} \mathcal{F}\left(\boldsymbol{x}, \boldsymbol{x}^{\prime}\right) H\left(\boldsymbol{x}, \boldsymbol{x}^{\prime}\right) \mathrm{d} V\left(\boldsymbol{x}^{\prime}\right) .
$$


This formulation suggests the definition of the integral length scale

$$
L_{\text {int }}^{2}(\boldsymbol{x})=\left|\int_{\Omega} \mathcal{F}\left(\boldsymbol{x}, \boldsymbol{x}^{\prime}\right) H\left(\boldsymbol{x}, \boldsymbol{x}^{\prime}\right) \mathrm{d} V\left(\boldsymbol{x}^{\prime}\right)\right|=\left|\frac{\rho \phi_{i j}^{*}(\boldsymbol{x})}{\Psi_{i j}(\boldsymbol{x}, \boldsymbol{x})}\right|,
$$

plotted in figure 13. Also plotted are the length scales used in Durbin's model (3.1), namely the Kolmogorov length scale $L_{K}$, which is active in the vicinity of the wall, and the turbulent length scale $L_{T}$.

The first important feature to be noted is that the correlation length scale is very close to the integral length scale in the main part of the flow, which leads to a very important conclusion: the most significant contribution of the correlation function to the integral (4.10) is given by its values at moderate separations. Integral properties of the correlation function are thus mainly due to the shape near the peak. The complex features of the correlation function demonstrated in figures 2 and 4-11, in particular the negative excursions, appear to have no significant influence. The redistribution term $\phi_{i j}^{*}$, which is given by the integral of the two-point correlation, can be expected to depend only on the shape of the correlation function at moderate separations. Therefore, the use of a simple model function, such as the exponential function (2.7), seems to be totally justified by figure 13 . However, as mentioned in $\S 4.2$, the asymmetry in the $y$-direction is missed by the model (2.7), which leads, as shown in Appendix B, to the erroneous amplification of the redistribution in the logarithmic layer noted in $\S 3.1$. The model must be modified, as shown in figure 3, to account for this asymmetry, as well as for the anisotropy emphasized in $\S 4.3$. This issue will be discussed in the following sections.

In the vicinity of the wall, the correlation length scale $L$ and the integral length scale $L_{\text {int }}$ do not have similar behaviours: $L$ approaches a value of 6 , whereas $L_{\text {int }}$ decreases rapidly toward the wall. This behaviour justifies the use of a lower bound in the model (3.1). The two length scales $L_{T}$ and $L_{K}$, used in this model, are also plotted in figure 13 . The turbulent length scale $L_{T}$ provides a very satisfactory representation of the integral length scale throughout the flow, except near the centre of the channel. It should be noted that in this region, both $\phi_{i j}^{*}$ and $\Psi_{i j}$ are small, and the evaluated integral scale is contaminated by numerical errors. Hence, the strong decrease of $L_{\text {int }}$ beyond $y^{+}=500$ is probably not physical. The curve has been truncated at $y^{+} \approx 550$ to improve the clarity of the figure. Since in the main part of the flow, the integral length scale $L_{i n t}$ and the correlation length scale $L$ are very close to each other, the model $L_{T}$ represents very satisfactorily the correlation length scale down to $y^{+} \approx 100$. Below this point, figure 13 shows that the Kolmogorov length scale $L_{K}$ must be used. The point where $L_{T}$ becomes smaller than $L_{K}$ when moving toward the wall is approximately located where $L$ and $L_{\text {int }}$ diverge. The location of this point depends on the coefficient $C_{\eta}$; in figure 13 , the original coefficient $C_{\eta}=80$, chosen to give the experimental value of the correlation length scale at the wall (Durbin \& Laurence 1996), has been used. In order to compare the shape of the modelled length scale against the data, the coefficient $C_{L}$ has been set to 0.045 in this figure. The fact that the length-scale shapes are very similar is an a posteriori justification for the use of the formulation $L=\max \left(L_{K}, L_{T}\right)$ in Durbin's model.

The above results have addressed some important issues about the elliptic relaxation method. In particular, they justify the use of a model for the correlation function which does not account for the negative excursions. They also confirm the necessity of bounding the length scale near the wall by the Kolmogorov length scale. However, it was shown in $\S 4.2$ and 4.3 that modelling the correlation function by a simple isotropic exponential function is too crude an approximation. This is at the origin of the spurious behaviour in the logarithmic layer. In the following sections, reformulations of the model will be proposed and their behaviour analyzed. 

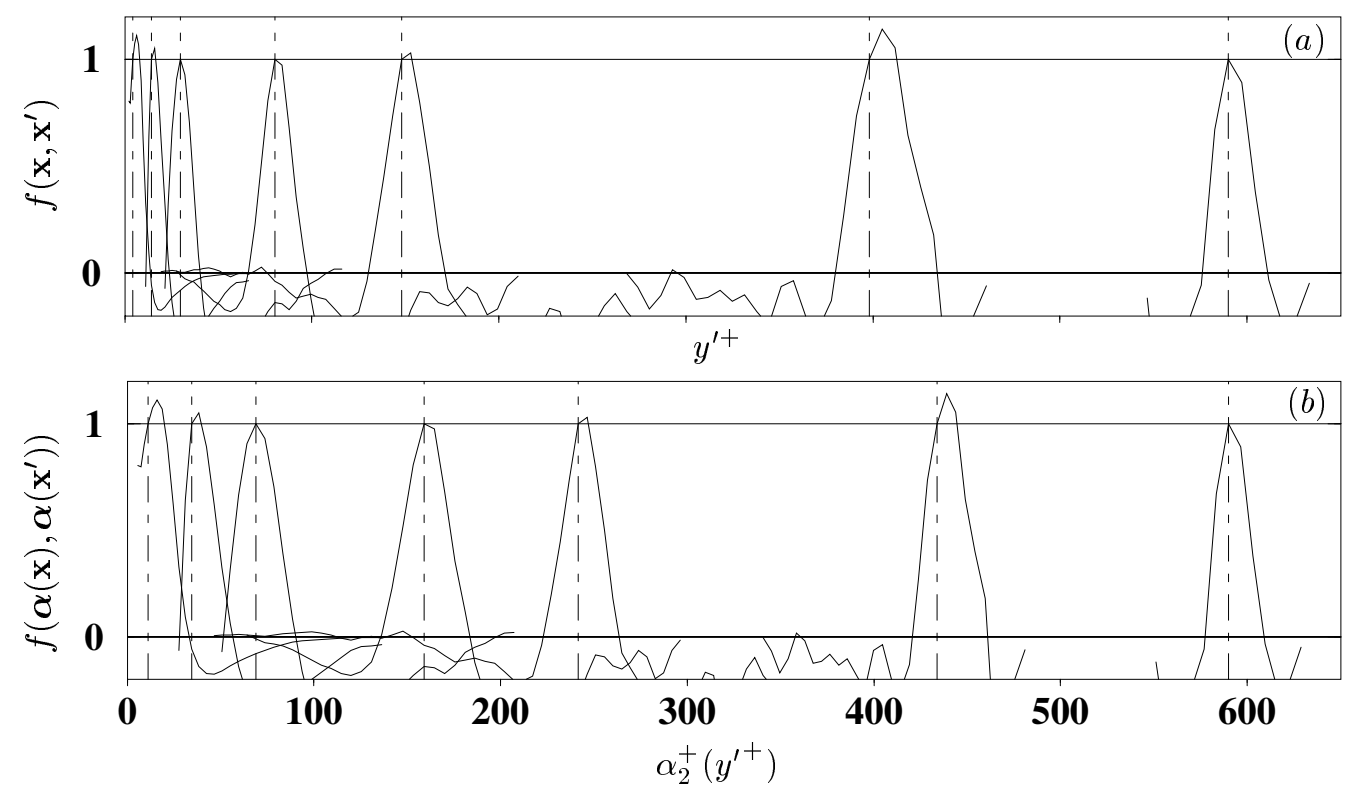

FIGURE 14. Effect of the space transformation on the correlation function: (a) Correlation function in the original space $\Omega$ (same as figure $2 b$ ); $(b)$ Correlation function after transformation of $y^{+}$-axis. See figure 2 for additional caption.

\section{Reformulation of the model}

\subsection{Correction of the inversion error}

The elliptic relaxation equation is derived from the integral equation of the redistribution term (2.4), which is transformed into (2.8) by using the model (2.7). Equation (2.8) can be inverted to give the elliptic relaxation equation if the integral is a convolution product, i.e., if the function denoted $E(r)$ truly depends only on $r$, or at most on the separation $\boldsymbol{x}^{\prime}-\boldsymbol{x}$. This is, however, not the case, since the length scale depends on the location. This inversion is valid only if the length scale can be considered locally constant, which implies that $\|\nabla L\|$ is much smaller than unity. Since the coefficient $C_{L}$ is chosen such that $\|\nabla L\|=\kappa=0.41$ in the logarithmic region, the length scale cannot be considered as a constant. If (2.9) is meant to be equivalent to (2.8), an inversion error is introduced.

In order to avoid this inversion error, it is necessary to treat the variation of $L$. A coordinate transformation $\boldsymbol{x} \mapsto \boldsymbol{\alpha}(\boldsymbol{x})$ can be introduced, such that in the transformed space the length scale is roughly a constant, and the boundaries of the domain are preserved: $\boldsymbol{\alpha}(\Omega)=\Omega$. In a channel, it is simply given implicitly by $\mathrm{d} \alpha_{2} / L_{\boldsymbol{\alpha}}=\mathrm{d} y / L(y)$, i.e., by the integral

$$
\alpha_{2}(y)=\int_{0}^{y} \frac{L_{\boldsymbol{\alpha}}}{L\left(y^{\prime}\right)} \mathrm{d} y^{\prime},
$$

where $L_{\boldsymbol{\alpha}}$ is a constant, chosen such that the domain is globally preserved:

$$
L_{\boldsymbol{\alpha}}=2 h\left(\int_{0}^{2 h} \frac{\mathrm{d} y^{\prime}}{L\left(y^{\prime}\right)}\right)^{-1} \text {. }
$$

Other directions can either be kept unchanged $\left(\alpha_{1}(\boldsymbol{x})=x\right.$ and $\left.\alpha_{3}(\boldsymbol{x})=z\right)$ or be transformed according to the previously noted anisotropy. An example of such a transformation in a 2-dimensional domain is given in appendix $\mathrm{C}$. 
Figure 14 shows how the shape of the correlation function corresponding to $\phi_{22}^{*}$ is modified by $\boldsymbol{\alpha}$. It can be seen that, after the transformation, the correlation length scale is constant across the channel. It can also be seen that the transformation almost completely removes the asymmetry in the $y$-direction. Note that, in this figure, the transformation of the correlation is plotted, instead of the correlation between transformed quantities (the space transformation and the evaluation of the two-point correlations are not commutable). This approximation is used only in this figure to illustrate the effect of $\boldsymbol{\alpha}$ on the length scale, but not in the subsequent analysis.

Let us define the functions: $\xi_{i}=\partial p / \partial x_{i} \circ \boldsymbol{\alpha}^{-1}, w_{i}=u_{i} \circ \boldsymbol{\alpha}^{-1}$ and $\zeta_{i j}=\phi_{i j}^{*} \circ \boldsymbol{\alpha}^{-1}$, where $\circ$ denotes the function composition: $f \circ g(\boldsymbol{x})=f(g(\boldsymbol{x}))$. Note that these functions are defined on the same domain $\Omega$ as the original quantities $\partial p / \partial x_{i}, u_{i}$ and $\phi_{i j}^{*}$, since $\boldsymbol{\alpha}$ maps $\Omega$ to itself. Additionally, let $g_{i}$ be the Laplacian of $\xi_{i}$, i.e.,

$$
g_{i}=\frac{\partial^{2} \xi_{i}}{\partial x_{k} \partial x_{k}} .
$$

The same method as in $\S 2$ can be applied to derive a new form of the elliptic relaxation equation. First, one can assume that a homogeneous Neumann boundary condition can be applied to $\xi_{k}$, so that it satisfies the following integral equation (see appendix $\mathrm{C}$ for details in a 1-dimensional case):

$$
\xi_{k}(\boldsymbol{x})=\int_{\Omega} g_{k}\left(\boldsymbol{x}^{\prime}\right) G_{\Omega}\left(\boldsymbol{x}, \boldsymbol{x}^{\prime}\right) \mathrm{d} V\left(\boldsymbol{x}^{\prime}\right) .
$$

In this expression, $G_{\Omega}$ is the same as in (2.3), since the Green function only depends on the domain. $\zeta_{i j}$ then satisfies

$$
\rho \zeta_{i j}(\boldsymbol{x})=\int_{\Omega} \Theta_{i j}\left(\boldsymbol{x}, \boldsymbol{x}^{\prime}\right) G_{\Omega}\left(\boldsymbol{x}, \boldsymbol{x}^{\prime}\right) \mathrm{d} V\left(\boldsymbol{x}^{\prime}\right),
$$

where $\Theta_{i j}\left(\boldsymbol{x}, \boldsymbol{x}^{\prime}\right)=-\overline{w_{j}(\boldsymbol{x}) g_{i}\left(\boldsymbol{x}^{\prime}\right)}-\overline{w_{i}(\boldsymbol{x}) g_{j}\left(\boldsymbol{x}^{\prime}\right)}$.

In a free space, (5.5) reduces to

$$
\rho \zeta_{i j}(\boldsymbol{x})=-\int_{\Omega} \Theta_{i j}\left(\boldsymbol{x}, \boldsymbol{x}^{\prime}\right) \frac{\mathrm{d} V\left(\boldsymbol{x}^{\prime}\right)}{4 \pi r} .
$$

The two-point correlations can then be modelled by

$$
\Theta_{i j}\left(\boldsymbol{x}, \boldsymbol{x}^{\prime}\right)=\Theta_{i j}\left(\boldsymbol{x}^{\prime}, \boldsymbol{x}^{\prime}\right) \exp \left(-\frac{r}{L_{\boldsymbol{\alpha}}}\right),
$$

leading to

$$
\rho \zeta_{i j}(\boldsymbol{x})=-\int_{\Omega} \Theta_{i j}\left(\boldsymbol{x}^{\prime}, \boldsymbol{x}^{\prime}\right) \frac{\exp \left(-\frac{r}{L_{\boldsymbol{\alpha}}}\right)}{4 \pi r} \mathrm{~d} V\left(\boldsymbol{x}^{\prime}\right) .
$$

Equation (5.8) is similar to (2.8), the main difference being that the length scale $L_{\boldsymbol{\alpha}}$ is now truly a constant. Thus, (5.8) is a convolution integral, which can be inverted without introducing an inversion error:

$$
\zeta_{i j}(\boldsymbol{x})-L_{\boldsymbol{\alpha}}^{2} \frac{\partial^{2} \zeta_{i j}}{\partial x_{k} \partial x_{k}}(\boldsymbol{x})=-\frac{L_{\boldsymbol{\alpha}}^{2}}{\rho} \Theta_{i j}(\boldsymbol{x}, \boldsymbol{x}) .
$$

Introducing the Jacobian matrix of the inverse transformation, $A=\nabla \alpha^{-1}$, the equation 
satisfied by $\phi_{i j}^{*}$ is

$$
\phi_{i j}^{*}-L_{\boldsymbol{\alpha}}^{2} A_{k l} A_{m l} \frac{\partial^{2} \phi_{i j}^{*}}{\partial x_{k} \partial x_{m}}-L_{\boldsymbol{\alpha}}^{2} A_{m l} \frac{\partial A_{k l}}{\partial x_{m}} \frac{\partial \phi_{i j}^{*}}{\partial x_{k}}=\phi_{i j}^{h} .
$$

In this equation, as in $\S 2.2$, the right hand side has been replaced by a quasi-homogeneous model, noting that in homogeneous situations, (5.10) reduces to

$$
\phi_{i j}^{*}=\phi_{i j}^{h} \text {. }
$$

Now, a matrix of length scales can be introduced by defining $A_{i j}=L_{i j} / L_{\boldsymbol{\alpha}}$, which yields a new form of the elliptic relaxation equation:

$$
\phi_{i j}^{*}-L_{k l} L_{m l} \frac{\partial^{2} \phi_{i j}^{*}}{\partial x_{k} \partial x_{m}}-L_{m l} \frac{\partial L_{k l}}{\partial x_{m}} \frac{\partial \phi_{i j}^{*}}{\partial x_{k}}=\phi_{i j}^{h} .
$$

This new formulation is more complicated than the original one, but allows for length scale anisotropy, which has been found to be very significant in $\S 4$. As will be shown in $\S 5.3$, it does not exhibit the same spurious behaviour in the logarithmic layer.

The remaining issue is the modelling of $L_{i j}$. The most natural choice is

$$
L_{i j}=\frac{3}{2} \frac{\overline{u_{i} u_{j}}}{k} L
$$

where $L$ is given by the original model (3.1). Another possibility is simply

$$
L_{i j}=L \delta_{i j}
$$

which neglects the anisotropy but reduces significantly the complexity of (5.12), which becomes

$$
\phi_{i j}^{*}-L^{2} \nabla^{2} \phi_{i j}^{*}-L \nabla L \cdot \nabla \phi_{i j}^{*}=\phi_{i j}^{h}
$$

This formulation only differs from the original one by the presence of the third term on the left hand side. This term accounts for the variations of the length scale. Note that (5.15) can be rewritten as

$$
\phi_{i j}^{*}-L \nabla \cdot\left(L \nabla \phi_{i j}^{*}\right)=\phi_{i j}^{h}
$$

This formulation is close to those proposed by Wizman et al. (1996), Laurence \& Durbin (1994) and Durbin \& Laurence (1996), but it will be demonstrated in $\S 5.3$ that it still exhibits an amplification of the redistribution in the logarithmic layer.

\subsection{Correction to the model of the correlation function}

In $\S 4.2$, it has been pointed out that the correlation function is strongly asymmetric in the direction normal to the wall. Figure 3 shows that using the original model for the correlation function, the two-point correlation between the fixed point $\boldsymbol{x}$ and the wall is overestimated. This shortcoming can be corrected by taking into account the gradient of the length scale, thus giving an asymmetric shape to the model for the correlation function:

$$
f\left(\boldsymbol{x}, \boldsymbol{x}^{\prime}\right)=\exp \left(\frac{-r}{L+\beta\left(\boldsymbol{x}^{\prime}-\boldsymbol{x}\right) \cdot \boldsymbol{\nabla} L}\right),
$$

which has been plotted for $\beta=1$ in figure 3 .

Considering the term $\beta\left(\boldsymbol{x}^{\prime}-\boldsymbol{x}\right) \cdot \boldsymbol{\nabla} L$ as a small correction, a Taylor series expansion of (5.17) leads to the expression

$$
f\left(\boldsymbol{x}, \boldsymbol{x}^{\prime}\right) \sim \exp \left(-\frac{r}{L}\right)\left(1+\beta \frac{r}{L^{2}}\left(\boldsymbol{x}^{\prime}-\boldsymbol{x}\right) \cdot \nabla L\right) .
$$


If (5.18) is combined with (2.6) and (2.4), using the free-space Green function, one obtains

$$
\begin{gathered}
\rho \phi_{i j}^{*}(\boldsymbol{x})=\underbrace{-\int_{\Omega} \Psi_{i j}\left(\boldsymbol{x}^{\prime}, \boldsymbol{x}^{\prime}\right) \frac{\exp \left(-\frac{r}{L}\right)}{4 \pi r} \mathrm{~d} V\left(\boldsymbol{x}^{\prime}\right)}_{\rho \phi_{i j}^{* a}} \\
\underbrace{-\int_{\Omega} \Psi_{i j}\left(\boldsymbol{x}^{\prime}, \boldsymbol{x}^{\prime}\right) \beta \frac{r}{L^{2}} \frac{\exp \left(-\frac{r}{L}\right)}{4 \pi r}\left(\boldsymbol{x}^{\prime}-\boldsymbol{x}\right) \cdot \boldsymbol{\nabla} L \mathrm{~d} V\left(\boldsymbol{x}^{\prime}\right)}_{\rho \phi_{i j}^{* b}} .
\end{gathered}
$$

In this equation, the first term, $\phi_{i j}^{* a}$, is exactly the redistribution term given by the original model, and satisfies

$$
\phi_{i j}^{* a}-L^{2} \nabla^{2} \phi_{i j}^{* a}=-\frac{L^{2}}{\rho} g_{i j},
$$

where $g_{i j}(\boldsymbol{x}) \equiv \Psi_{i j}(\boldsymbol{x}, \boldsymbol{x})$. The second term $\phi_{i j}^{* b}$ can be considered as a correction term. Using a Taylor series expansion of $g_{i j}\left(\boldsymbol{x}^{\prime}\right)$ in the vicinity of the fixed point $\boldsymbol{x}$, one obtains

$$
\rho \phi_{i j}^{* b}(\boldsymbol{x})=-\int_{\Omega}\left(\boldsymbol{x}^{\prime}-\boldsymbol{x}\right) \cdot \nabla g_{i j} \beta \frac{r}{L^{2}}\left(\boldsymbol{x}^{\prime}-\boldsymbol{x}\right) \cdot \boldsymbol{\nabla} L \frac{\exp \left(-\frac{r}{L}\right)}{4 \pi r} \mathrm{~d} V\left(\boldsymbol{x}^{\prime}\right),
$$

which gives exactly, in a free space,

$$
\rho \phi_{i j}^{* b}=-8 \beta L^{3} \nabla L \cdot \nabla g_{i j} .
$$

Following Durbin (1991), the right hand side of (5.20) can be replaced by any quasihomogeneous model $\phi_{i j}^{h}$, which corresponds to modelling $g_{i j}$ by $-\rho \phi_{i j}^{h} / L^{2}$. There are then two possible ways to take into account the correction term in the model. First, in (5.22), $g_{i j}$ can be approximated by $-\rho \phi_{i j}^{* a} / L^{2}$, which leads to

$$
\begin{gathered}
\phi_{i j}^{* a}-L^{2} \nabla^{2} \phi_{i j}^{* a}=\phi_{i j}^{h} ; \\
\rho \phi_{i j}^{* b}=8 \beta L \nabla L \cdot \nabla \phi_{i j}^{* a}-16 \beta(\nabla L)^{2} \phi_{i j}^{* a} .
\end{gathered}
$$

Thus, (5.23) gives exactly the same solution as the original model, while (5.24) provides an explicit correction $\left(\phi_{i j}^{*}=\phi_{i j}^{* a}+\phi_{i j}^{* b}\right)$. The second possibility is to take into account the correction implicitly:

$$
\left(1+16 \beta(\nabla L)^{2}\right) \phi_{i j}^{*}-L^{2} \nabla^{2} \phi_{i j}^{*}-8 \beta L \nabla L \cdot \nabla \phi_{i j}^{*}=\phi_{i j}^{h} .
$$

This implicit formulation is probably preferable for stability reasons. In (5.25), the same term $L \boldsymbol{\nabla} L \cdot \boldsymbol{\nabla} \phi_{i j}^{*}$ as in (5.15) appears, but with the coefficient $8 \beta$. This shows that the space transformation introduced in $\S 5.1$ also corrects the asymmetry in the $y$-direction, as can be seen in figure 14 .

It is worth pointing out that the same type of correction has been used by Launder \& Tselepidakis (1991), in order to take into account the inhomogeneity of the flow near a wall, and thus avoid the use of wall echo correction terms. Usually, the rapid part of the redistribution term is evaluated as the tensorial product between a fourth order tensor and the gradient of the mean velocity. Launder \& Tselepidakis (1991) proposed to replace 


\begin{tabular}{|c|c|c|c|}
\hline Equation & Model & $\gamma$ & $\Gamma$ \\
\hline$(2.10)$ & D1 (Durbin 1991) & -2 & 1.51 \\
\hline$(3.3)$ & W1 (Wizman et al. 1996) & 0 & 1 \\
\hline$(3.5)$ & L1 (Laurence \& Durbin 1994) & 0 & 1 \\
\hline (3.6) & D2 (Durbin \& Laurence 1996) & 0 & 1 \\
\hline$(3.4)$ & W2 (Wizman et al. 1996) & 2 & 0.75 \\
\hline$(5.15)$ & M1 (this paper) & -1 & 1.2 \\
\hline$(5.12)$ & M2 (this paper) & $-\frac{9}{4}\left({\overline{u_{1} u_{2}}}^{2} / k^{2}+{\overline{u_{2} u_{2}}}^{2} / k^{2}\right)$ & 1.06 \\
\hline$(5.25)$ & M3 (this paper) & $2(12 \beta-1)$ & $0-1.51$ \\
\hline$(5.29)$ & GS (Gibson \& Launder 1978) & NA & 0.67 \\
\hline$(5.30)$ & GR (Gibson \& Launder 1978) & NA & 0.40 \\
\hline$(5.26)$ & LT (Launder \& Tselepidakis 1991) & NA & 0.53 \\
\hline
\end{tabular}

the latter by an effective velocity gradient given by

$$
\left.\frac{\partial U_{l}}{\partial x_{m}}\right|_{\mathrm{eff}}=\frac{\partial U_{l}}{\partial x_{m}}+c_{I} l_{n} \frac{\partial l_{n}}{\partial x_{k}} \frac{\partial^{2} U_{l}}{\partial x_{k} \partial x_{m}}
$$

where $c_{I}=0.3$, and $l_{n}=(k / \varepsilon)\left(\overline{u_{p} u_{q}} n_{p} n_{q}\right)^{1 / 2}$ is a scalar length scale in the direction of outward normal to the wall. Thus, in (5.15), (5.25) and (5.26), by three different reasonings, the same type of correction, of the form $L \nabla L \cdot \nabla A$, where $A$ denotes either $\phi_{i j}^{*}$ or $\partial U_{l} / \partial x_{m}$, has been introduced in order to reduce the energy redistribution between the components of the Reynolds stress in the logarithmic layer. The effect of the corrections proposed in the present paper is investigated in the next section.

\subsection{Redistribution reduction in the logarithmic layer}

In $\S 3.1$, it has been shown that, irrespective of the quasi-homogeneous model, the original formulation gives an amplification of the redistribution in the logarithmic layer. In fact, no matter what formulation of the elliptic relaxation equation is considered, the same analysis leads to an expression of the form

$$
\phi_{i j}^{*}=\Gamma \phi_{i j}^{h}
$$

where

$$
\Gamma=\frac{1}{1+\gamma C_{L}^{2} C_{\mu}^{-3 / 2} \kappa^{2}},
$$

thus relating the redistribution term given by the elliptic relaxation model to its underlying quasi-homogeneous model. This analysis is based on the logarithmic layer assumptions: $\phi_{i j}^{h}=A^{h} / y, k=u_{\tau}^{2} / C_{\mu}^{1 / 2}$ and $\varepsilon=u_{\tau}^{3} / \kappa y$, where $A^{h}$ is a constant and $u_{\tau}$ denotes the friction velocity. In $(5.27), \phi_{i j}^{*}$ is not the exact solution of the differential equation, but only a particular solution in the logarithmic layer (no boundary conditions are considered). The amplification factor $\Gamma$ characterizes the effect of the elliptic relaxation equation in this region.

The coefficient $\gamma$ in (5.28) depends on the formulation of the elliptic relaxation equa- 
tion. It is given in table 1 , along with an estimation of the amplification factor $\Gamma$. The length scale $L$ used in the calculations is given by (3.1) for all the formulations. No alternative definition of $L$ is needed in the new formulations M1, M2 and M3, since they explicitly involve the gradient of $L$ to account for inhomogeneity. The coefficient $C_{L}$ is chosen such that the length scale is $L=\kappa y$ in the logarithmic layer: $C_{L}=C_{\mu}^{3 / 4}$. In the case of the fully anisotropic model M2, the anisotropies $a_{i j}=\overline{u_{i} u_{j}} / k-\frac{2}{3} \delta_{i j}$ in the logarithmic layer are required, since they enter the anisotropic length scale (5.13). The following logarithmic layer values have been used: $a_{12}=-0.30 ; a_{22}=-0.42$. In the case of model M3, $\gamma$, and hence $\Gamma$, are functions of the coefficient $\beta$. As $\beta$ varies from 0 (original model D1) to $\infty, \Gamma$ varies from 1.51 to 0 . Note that the explicit version of M3, given by (5.23) and (5.24), has the same behaviour as M3 to the first order.

It can be seen in the table that the new models M1, M2 and M3, which are derived from theoretical considerations, unlike the ad hoc models W1, L1, D2 and W2, have various behaviours. The first one, M1, which attempts to limit the inversion error ( $\$ 5.1$ ) but does not account for the anisotropy of the length scale, induces an amplification of the redistribution. The factor $\Gamma_{\mathrm{M} 1}$ is lower than $\Gamma_{\mathrm{D} 1}$, since the term $-L \nabla L \cdot \nabla \phi_{i j}^{*}$, which distinguishes M1 from D1, moves in the right direction. However, it is not sufficiently large to remove all the amplification due to the term $L^{2} \nabla^{2} \phi_{i j}^{*}$. When the length scale anisotropy is taken into account (M2), the amplification factor falls to $\Gamma_{\mathrm{M} 2}=1.06$. Thus, model M2 can be referred to as a quasi-neutral model. In model M3, $\beta$ can be chosen to give any amplification factor between 0 and 1.51. The presence of the coefficient $\beta$ enables adjustment of the weights of the terms $-L \nabla L \cdot \nabla \phi_{i j}^{*}$ and $(\boldsymbol{\nabla} L)^{2} \phi_{i j}^{*}$. By choosing $\beta=\frac{1}{12}$, the model becomes neutral $(\Gamma=1)$.

The amplification factors for the 22-components of the slow and rapid parts of the Gibson \& Launder (1978) model are shown in table 1 for comparison. The slow part of the wall echo terms is given by

$$
\phi_{i j}^{w S}=C_{1}^{\prime} \frac{\varepsilon}{k}\left(\overline{u_{k} u_{m}} n_{k} n_{m} \delta_{i j}-\frac{3}{2} \overline{u_{k} u_{i}} n_{k} n_{j}-\frac{3}{2} \overline{u_{k} u_{j}} n_{k} n_{i}\right) f\left(\frac{L_{T}}{n_{i} r_{i}}\right),
$$

where $C_{1}^{\prime}=0.5$. The rapid part is

$$
\phi_{i j}^{w R}=C_{2}^{\prime}\left(\phi_{k m} n_{k} n_{m} \delta_{i j}-\frac{3}{2} \phi_{i k} n_{k} n_{j}-\frac{3}{2} \phi_{j k} n_{k} n_{i}\right) f\left(\frac{L_{T}}{n_{i} r_{i}}\right),
$$

where $C_{2}^{\prime}=0.3$. The amplification factor for the formulation (5.26) proposed by Launder \& Tselepidakis (1991), used with the linear IP model, is also shown.

This simple analysis of the logarithmic layer shows that modifications of the elliptic relaxation equation can overcome the deficiencies of the original model. The formulation to be chosen depends on the quasi-homogeneous model used as the source term. The most natural choice is to use a model which predicts correctly the redistribution in the logarithmic layer, such as the Speziale, Sarkar \& Gatski (1991) model (see, e.g., Demuren \& Sarkar 1993; Hadžić 1999; Manceau 1999), together with a neutral formulation of the elliptic relaxation equation, M2 or M3 with $\beta=\frac{1}{12}$. Less elaborate models, such as the Rotta \& IP model, which overestimates the redistribution in the logarithmic layer, should be used with a formulation like the M3 model, with a coefficient $\beta$ larger than $\frac{1}{12}$, without any wall echo correction terms.

Since these new formulations of the elliptic relaxation equation have amplification factors close to those previously proposed by Wizman et al. (1996), Laurence \& Durbin (1994) and Durbin \& Laurence (1996), they are expected to give similar results in practi- 
cal applications. It was noted, in $\S 3.1$, that the formulations W1, L1, D2 and W2 suffer from a lack of justification, and accordingly, the new formulations should be preferred.

Further a priori tests and actual computations using the new formulation M3 have been performed recently. The results, discussed in detail elsewhere (Manceau \& Hanjalić 2000), demonstrate that an overall benefit is obtained by using the modified form of the elliptic relaxation equation in a channel flow. The a priori tests show that when the Rotta \& IP model is used as the source term of the elliptic relaxation equation, the M3 model, with the coefficient $\beta$ chosen to obtain a reduction of the redistribution in the logarithmic layer, corrects the overestimation due to the Rotta \& IP model as expected. The results are comparable to those obtained from the redistribution-reducing model W2. If the SSG model (Speziale et al. 1991), which correctly predicts the redistribution in the logarithmic layer, is used as the source term, the M3 model gives results similar to those from the neutral W1 model. Both models are shown to be preferable to the original (D1) model.

Consequently, the optimal combinations of the SSG model (as source term) with the neutral elliptic relaxation formulations W1 and M3, respectively, are implemented in a Reynolds stress model and tested in full computations of a channel flow (Manceau \& Hanjalic 2000). The results given by the two forms W1 and M3 are nearly indistinguishable. The use of these neutral formulations improves, in the buffer and logarithmic layers, the prediction of the mean velocity profile, which is underpredicted by the original elliptic relaxation model. Moreover, the anisotropy, and in particular the peak of $\overline{u^{2}}$, are better captured by these models.

Manceau \& Hanjalić (2000) further show that, in the framework of the $\overline{v^{2}}-f$ model (Durbin 1991), the two neutral models (W1 and M3 with $\beta=\frac{1}{12}$ ) as well as a redistribution-reducing model (M3 with $\beta=\frac{1}{6}$ ) are capable of correcting the overestimation of the mean velocity by the original formulation, without adversely affecting the prediction of the turbulent kinetic energy.

\section{Conclusions}

The elliptic relaxation method is a promising way to model correctly the redistribution term down to solid boundaries. It can be expected to be somewhat universal, since it is based on theoretical grounds. However, some of the modelling assumptions can be called into question, and the behaviour of the original model is not entirely satisfactory in the logarithmic layer. The DNS database of a channel flow at $R e_{\tau}=590$ (Moser et al. 1999) has been analyzed in order to understand the reasons for this spurious behaviour and to examine a number of open issues. Several conclusions can be drawn:

- The use of an exponential form to model the correlation function between the velocity and the Laplacian of the pressure gradient is consistent with the data. In particular, the coincidence of the integral scale and the half width of the correlation function for moderate separations shows that the correlation function shape for large separations does not have a significant influence on the redistribution term.

- The presence of a solid boundary induces a strong anisotropy of the turbulent structures, and in particular a significant asymmetry in the wall-normal direction. This feature is not accounted for by the simple isotropic correlation function used in Durbin's model. This weakness is at the origin of the erroneous amplification of the redistribution of energy between the components of the Reynolds stress observed in the logarithmic layer.

- The modelling of the correlation length scale by the turbulent length scale bounded near the wall by the Kolmogorov length scale is well corroborated by the DNS data.

- The wall echo effect, which corresponds to the appearance of image terms in the 
Green function, increases the redistribution of energy, contrary to the common belief. Accordingly, it cannot be considered responsible for the observed damping in the logarithmic layer.

This study shows that the elliptic relaxation model, first derived intuitively by Durbin (1991), is based on assumptions relatively consistent with the DNS database. Even though the model is too simple to represent exactly the two-point correlations involved in the integral equation of the redistribution term, it reproduces the important non-local effect which cannot be accounted for by any algebraic model.

Based on the physical insights gained through the present DNS analysis, modifications of the model have been proposed in order to account for the inhomogeneity and anisotropy effects. The first modification is based on the observation that the length scale in the elliptic relaxation equation cannot be considered locally as constant. A new formulation of this equation, which accounts for this feature, includes the effect of the anisotropy of turbulence through a rather complicated tensorial expression. In the second modification, an asymmetric correlation function is introduced, by using the gradient of the length scale to identify the direction of inhomogeneity. This results in a second new formulation of the elliptic relaxation equation, involving fewer terms than the first one.

Both new formulations do not exhibit the same amplification of the redistribution in the logarithmic layer as the original one. The first one can be referred to as quasi-neutral since its amplification factor is only $\Gamma=1.06$, compared to $\Gamma=1.51$ for the original formulation. The second formulation can be neutral or exhibit a reduction, depending on the coefficient $\beta$ which controls the sensitivity of the correlation function to the length scale gradient. Thus, either one of the formulations can be chosen, depending on the quasi-homogeneous model used as the source term and whether or not its behaviour in the logarithmic layer needs correction. The new formulations are similar to those proposed by Wizman et al. (1996), Laurence \& Durbin (1994) and Durbin \& Laurence (1996), but have the advantage of being based on rigorous theoretical grounds.

Further studies, including a priori tests and computations in a channel flow using new formulations, have been conducted (Manceau \& Hanjalić 2000). It has been shown that modifying the elliptic operator according to (5.25) indeed improves, in the logarithmic region, the mean velocity profile and the turbulent quantities predicted by Durbin's Reynolds stress model as well as by the $\overline{v^{2}}-f$ model. In future studies, the new formulations will be tested and calibrated in more canonical test cases, in order to be ultimately applicable to complex engineering flows.

The authors gratefully acknowledge Paul Durbin and Joel Ferziger for the valuable comments on a draft of this manuscript, and William Cabot, Javier Jimenez and Robert Moser for assistance with the DNS database and useful discussions. R. M. acknowledges the hospitality of the Center for Turbulence Research during the 1998 summer research program. M. W. acknowledges support by the U.S. Office of Naval Research under Grant N00014-95-1-0221.

\section{Appendix A. Approximation of the Green function in a channel}

The purpose of this appendix is to derive an approximate Green function in a channel that is sufficient to preserve the correct features of the solution of a Neumann problem, and in particular its boundary conditions.

Let us consider the channel $\mathcal{C}_{0}=\mathbb{R} \times[0,1] \times \mathbb{R}$ shown in figure 15 , bounded by two infinite planes $\mathcal{P}_{0}$ and $\mathcal{P}_{1}$ located in $y=0$ and $y=1$, respectively. In this domain, the 

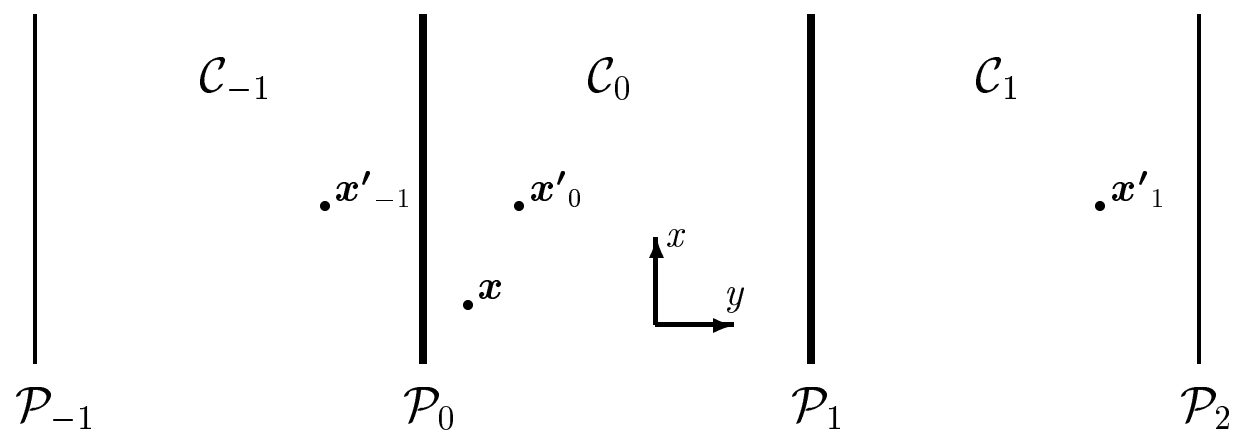

Figure 15 . Sketch of the channel $\mathcal{C}_{0}=\mathbb{R} \times[0,1] \times \mathbb{R}$ and its images $\mathcal{C}_{-1}$ and $\mathcal{C}_{1}$

problem to be solved is

$$
\left\{\begin{array}{l}
\nabla^{2} f=g, \\
\frac{\partial f}{\partial \boldsymbol{n}}=0 \text { on } \partial \mathcal{C}_{0}=\mathcal{P}_{0} \cup \mathcal{P}_{1},
\end{array}\right.
$$

where $\boldsymbol{n}$ is the outgoing unit vector normal to the wall. The general solution of this Neumann problem is given by

$$
f(\boldsymbol{x})=-\int_{\mathcal{C}_{0}} \frac{g\left(\boldsymbol{x}^{\prime}\right)}{4 \pi\left\|\boldsymbol{x}^{\prime}-\boldsymbol{x}\right\|} \mathrm{d} V\left(\boldsymbol{x}^{\prime}\right)-\int_{\partial \mathcal{C}_{0}} f\left(\boldsymbol{x}^{\prime}\right) \frac{\partial}{\partial \boldsymbol{n}^{\prime}}\left(\frac{1}{4 \pi\left\|\boldsymbol{x}^{\prime}-\boldsymbol{x}\right\|}\right) \mathrm{d} S\left(\boldsymbol{x}^{\prime}\right) .
$$

If the Green function of the channel, which satisfies the Neumann boundary condition on $\mathcal{P}_{0}$ and $\mathcal{P}_{1}$, is used, the surface integral vanishes, leading to

$$
f(\boldsymbol{x})=\int_{\mathcal{C}_{0}} g\left(\boldsymbol{x}^{\prime}\right) G_{\mathcal{C}_{0}}\left(\boldsymbol{x}, \boldsymbol{x}^{\prime}\right) \mathrm{d} V\left(\boldsymbol{x}^{\prime}\right)
$$

Unfortunately, no simple analytical expression of $G_{\mathcal{C}_{0}}$ exists except in the $\left(k_{x}, k_{z}\right)$ wavenumber space. Therefore, it is necessary to derive an approximated form of the Green function in the physical space.

We denote $H\left(\boldsymbol{x}, \boldsymbol{x}^{\prime}\right)$ as the approximate Green function which renders the surface term in (A 2) negligibly small. Chou (1945) proposed to let $H=G_{\mathbf{R}^{3}}$, the free-space Green function, but noted that it is only valid far from solid boundaries.

Let us consider the image channels $\mathcal{C}_{-1}$ and $\mathcal{C}_{1}$ shown in figure (15), which are symmetrical to $\mathcal{C}_{0}$ with respect to $\mathcal{P}_{0}$ and $\mathcal{P}_{1}$, respectively. The "even" extension $\tilde{g}$ of $g$ can be defined by $\tilde{g}\left(\boldsymbol{x}_{0}^{\prime}\right)=\tilde{g}\left(\boldsymbol{x}^{\prime}{ }_{-1}\right)=\tilde{g}\left(\boldsymbol{x}_{1}{ }_{1}\right)=g\left(\boldsymbol{x}_{0}{ }_{0}\right)$ for each point $\boldsymbol{x}_{0}{ }_{0}$ of the channel $\mathcal{C}_{0}$, where the points $\boldsymbol{x}^{\prime}{ }_{-1}$ and $\boldsymbol{x}^{\prime}{ }_{1}$ are symmetrical to $\boldsymbol{x}^{\prime}{ }_{0}$ with respect to $\mathcal{P}_{0}$ and $\mathcal{P}_{1}$, respectively.

Then, $\tilde{f}$, the extension of $f$, is a solution of a Neumann problem in $\mathcal{C}_{-1} \cup \mathcal{C}_{0} \cup \mathcal{C}_{1}$ :

$$
\left\{\begin{array}{l}
\nabla^{2} \tilde{f}=\tilde{g}, \\
\frac{\partial \tilde{f}}{\partial \boldsymbol{n}}=0 \text { on } \quad \mathcal{P}_{-1} \cup \mathcal{P}_{2} .
\end{array}\right.
$$


The solution can be expressed as

$$
\begin{gathered}
\tilde{f}(\boldsymbol{x})=-\int_{\mathcal{C}_{-1} \cup \mathcal{C}_{0} \cup \mathcal{C}_{1}} \frac{\tilde{g}\left(\boldsymbol{x}^{\prime}\right)}{4 \pi\left\|\boldsymbol{x}^{\prime}-\boldsymbol{x}\right\|} \mathrm{d} V\left(\boldsymbol{x}^{\prime}\right) \\
-\int_{\mathcal{P}_{2}} \frac{\partial}{\partial y^{\prime}}\left(\frac{1}{4 \pi\left\|\boldsymbol{x}^{\prime}-\boldsymbol{x}\right\|}\right) \tilde{f}\left(\boldsymbol{x}^{\prime}\right) \mathrm{d} S\left(\boldsymbol{x}^{\prime}\right)+\int_{\mathcal{P}_{-1}} \frac{\partial}{\partial y^{\prime}}\left(\frac{1}{4 \pi\left\|\boldsymbol{x}^{\prime}-\boldsymbol{x}\right\|}\right) \tilde{f}\left(\boldsymbol{x}^{\prime}\right) \mathrm{d} S\left(\boldsymbol{x}^{\prime}\right) .
\end{gathered}
$$

By splitting the volume integral in (A 5) into integrals on $\mathcal{C}_{-1}, \mathcal{C}_{0}$ and $\mathcal{C}_{1}$, adding and subtracting surface integrals on $\mathcal{P}_{0}$ and $\mathcal{P}_{1}$ and using the identities $\tilde{f}\left(\boldsymbol{x}^{\prime}{ }_{-1}\right)=\tilde{f}\left(\boldsymbol{x}^{\prime}{ }_{1}\right)=$ $f\left(\boldsymbol{x}_{0}^{\prime}\right)$ and $\tilde{g}\left(\boldsymbol{x}_{-1}^{\prime}\right)=\tilde{g}\left(\boldsymbol{x}_{1}^{\prime}\right)=g\left(\boldsymbol{x}_{0}^{\prime}\right)$, it can be easily shown that:

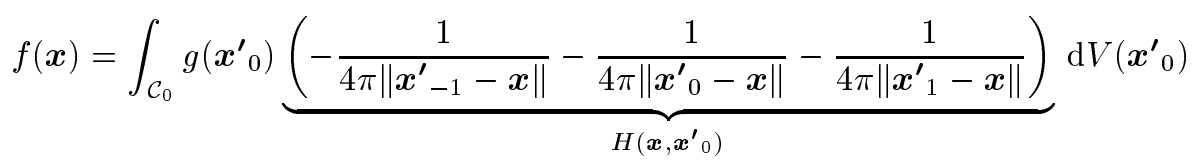

$$
\begin{aligned}
& +\int_{\partial \mathcal{C}_{0}} f\left(\boldsymbol{x}^{\prime}{ }_{0}\right) \frac{\partial}{\partial \boldsymbol{n}^{\prime}}\left(-\frac{1}{4 \pi\left\|\boldsymbol{x}_{-1}^{\prime}-\boldsymbol{x}\right\|}-\frac{1}{4 \pi\left\|\boldsymbol{x}_{0}^{\prime}-\boldsymbol{x}\right\|}-\frac{1}{4 \pi\left\|\boldsymbol{x}_{1}{ }_{1}-\boldsymbol{x}\right\|}\right) \mathrm{d} S\left(\boldsymbol{x}^{\prime}{ }_{0}\right) .
\end{aligned}
$$

The surface integral in this expression can now be neglected. Indeed, the derivative of $H\left(\boldsymbol{x}, \boldsymbol{x}_{0}^{\prime}\right)$ is

$$
\frac{\partial}{\partial \boldsymbol{n}^{\prime}} H\left(\boldsymbol{x}, \boldsymbol{x}_{0}^{\prime}\right)=-\frac{\left(\boldsymbol{x}_{-1}-\boldsymbol{x}\right) \cdot \boldsymbol{n}^{\prime}}{4 \pi\left\|\boldsymbol{x}_{-1}-\boldsymbol{x}\right\|^{3}}+\frac{\left(\boldsymbol{x}_{0}^{\prime}-\boldsymbol{x}\right) \cdot \boldsymbol{n}^{\prime}}{4 \pi\left\|\boldsymbol{x}_{0}^{\prime}-\boldsymbol{x}\right\|^{3}}-\frac{\left(\boldsymbol{x}_{1}^{\prime}-\boldsymbol{x}\right) \cdot \boldsymbol{n}^{\prime}}{4 \pi\left\|\boldsymbol{x}_{1}^{\prime}-\boldsymbol{x}\right\|^{3}} .
$$

On $\mathcal{P}_{0}$, since $\boldsymbol{x}^{\prime}{ }_{-1}=\boldsymbol{x}^{\prime}{ }_{0}$, the sum of the first two terms is zero and the surface integral only involves the contribution of the image $\boldsymbol{x}^{\prime}{ }_{1}$. To estimate its magnitude, we choose a function $f$ such that $f(x, 0, z)=f_{0} \chi(x, z)$, where $\chi(x, z)=1$ for $x^{2}+z^{2} \leqslant R^{2}$ and 0 elsewhere. The contribution $f_{\mathcal{P}_{0}}$ of the surface integral on $\mathcal{P}_{0}$ is

$$
f_{\mathcal{P}_{0}}=\frac{1}{2} f_{0}\left(1-\frac{2-y}{\left(R^{2}+(2-y)^{2}\right)^{1 / 2}}\right),
$$

which takes the value

$$
f_{\mathcal{P}_{0}}=f_{0}\left(\frac{1}{2}-\frac{1}{\left(R^{2}+2^{2}\right)^{1 / 2}}\right)
$$

at $y=0$. This value can be very small, depending on $R$.

In the case of the redistribution term, the two-point correlation $\overline{u_{i}(\boldsymbol{x}) \partial p / \partial x_{j}\left(\boldsymbol{x}^{\prime}\right)}+$ $\overline{u_{j}(\boldsymbol{x}) \partial p / \partial x_{i}\left(\boldsymbol{x}^{\prime}\right)}$ in the surface term can be approximated by

$$
\left(\overline{u_{i}(\boldsymbol{x}) \frac{\partial p}{\partial x_{j}}(\boldsymbol{x})}+\overline{u_{j}(\boldsymbol{x}) \frac{\partial p}{\partial x_{i}}(\boldsymbol{x})}\right) \chi\left(x^{\prime}-x, z^{\prime}-z\right) .
$$

The radius $R$ defining $\chi$ is the non-dimensional correlation length scale $L / 2 h$, where $h$ is the half-width of the channel. If the $\phi_{22}^{*}$ component is considered, the correlation length scale at the wall, evaluated from DNS data, is at most $L^{+}=25$ (in the streamwise direction), as can be seen in figure 12 . With this value, $R \approx 0.02$. The Taylor series expansion with respect to $R$ of the surface contribution $f_{\mathcal{P}_{0}}=\phi_{i j_{\mathcal{P}_{0}}}$, given by (A 9), leads to

$$
\phi_{i j \mathcal{P}_{0}}^{*} \sim \frac{L^{2}}{64 h^{2}} \phi_{i j}^{*} \approx 3 \times 10^{-5} \phi_{i j}^{*} .
$$

This result shows that the present approximation of the Green function is much better 
than $G_{\mathbb{R}^{3}}$, which gives a factor of 0.5 . Moreover, the boundary condition of $f$ at the wall will be much more correctly imposed with this approximation.

One could think that by the same argument, adding more and more image terms would lead to the vanishing of the surface integral and that the Green function of the channel is exactly the sum of an infinity of image terms. Unfortunately, this sum is not convergent so the exact Green function cannot be derived this way.

\section{Appendix B. Consequence of symmetrizing the correlation function}

The purpose of this appendix is to provide a formal proof that the value of the integral (the redistribution term in our case) is increased when the correlation function is symmetrized.

Let us define functions $g(\boldsymbol{x})$ (corresponding to $\Psi(\boldsymbol{x}, \boldsymbol{x})$ in this paper) and $h\left(\boldsymbol{x}, \boldsymbol{x}^{\prime}\right)$ $\left(f\left(\boldsymbol{x}, \boldsymbol{x}^{\prime}\right) G_{\Omega}\left(\boldsymbol{x}, \boldsymbol{x}^{\prime}\right)\right.$ in this paper $)$, and the integral

$$
I(\boldsymbol{x})=\int_{\Omega} g\left(\boldsymbol{x}^{\prime}\right) h\left(\boldsymbol{x}, \boldsymbol{x}^{\prime}\right) \mathrm{d} V\left(\boldsymbol{x}^{\prime}\right)
$$

which corresponds to $\phi_{i j}^{*}(\boldsymbol{x})$. If $x$ and $z$ are the homogeneous directions, $h\left(\boldsymbol{x}, \boldsymbol{x}^{\prime}\right)=$ $h\left(x^{\prime}-x, y, y^{\prime}, z^{\prime}-z\right)$ and $g(\boldsymbol{x})=g(y)$. The integral $I(\boldsymbol{x})$ then reduces to

$$
I(y)=\int_{y} g\left(y^{\prime}\right) H\left(y, y^{\prime}\right) \mathrm{d} y^{\prime}
$$

where $H\left(y, y^{\prime}\right)=\int_{x} \int_{z} h\left(x^{\prime}-x, y, y^{\prime}, z^{\prime}-z\right) \mathrm{d} x^{\prime} \mathrm{d} z^{\prime}$.

Two assumptions, consistent with the behaviour of the correlation functions in this paper, are used in the following derivation: $H\left(y, y^{\prime}\right)$ decreases faster when $y^{\prime}<y$ than when $y^{\prime}>y(H(y, y+s)>H(y, y-s)$ for $s>0)$, and $g(y)$ decreases when $y$ increases.

Let us now define $H^{*}\left(y, y^{\prime}\right)$ by symmetrizing $H\left(y, y^{\prime}\right)$ :

$$
H^{*}(y, y+s)=\frac{H(y, y-s)+H(y, y+s)}{2}
$$

for all $s$. The integral $I^{*}(y)$ is simply defined by replacing $H\left(y, y^{\prime}\right)$ by $H^{*}\left(y, y^{\prime}\right)$ in (B 2). The objective now is to show that $I^{*}(y)-I(y)>0$.

The function $I^{*}(y)$ can be split into its left and right parts:

$$
I^{*}(y)=\int_{s>0}\left[g(y-s) H^{*}(y, y-s)+g(y+s) H^{*}(y, y+s)\right] \mathrm{d} s .
$$

Using the definition of $H^{*}\left(y, y^{\prime}\right)$, this can be written as

$$
I^{*}(y)=\int_{s>0}[g(y-s)+g(y+s)] \frac{H(y, y-s)+H(y, y+s)}{2} \mathrm{~d} s .
$$

On the other hand, $I(y)$ can be split into left and right parts as well:

$$
I(y)=\int_{s>0}[g(y-s) H(y, y-s)+g(y+s) H(y, y+s)] \mathrm{d} s,
$$

and thus, a combination of (B 5) and (B 6) yields:

$$
I^{*}(y)-I(y)=\frac{1}{2} \int_{s>0}[g(y-s)-g(y+s)][H(y, y+s)-H(y, y-s)] \mathrm{d} s .
$$

Based on the assumptions about $g(y)$ and $H\left(y, y^{\prime}\right)$ made earlier, both terms in the square brackets are positives and, accordingly, $I^{*}(y)-I(y)>0$. 
(a)

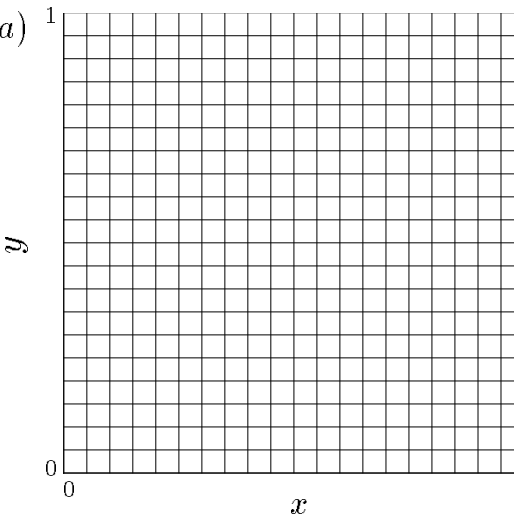

(b)
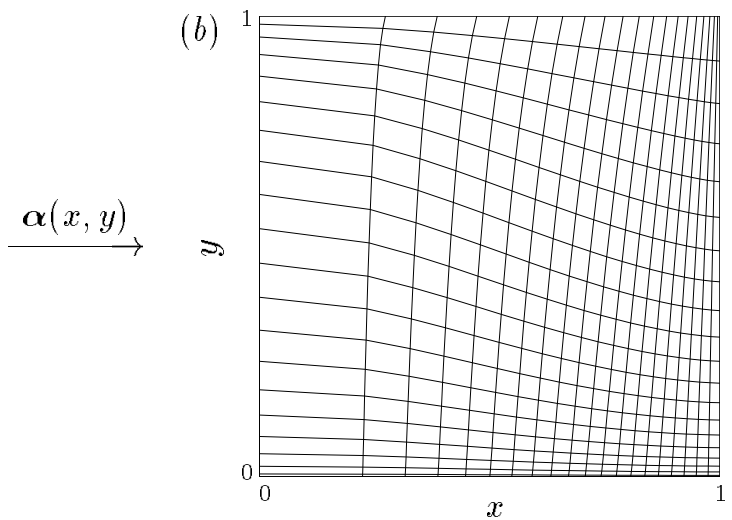

Figure 16. Example of mapping a $[0,1] \times[0,1]$ domain to itself. Lines drawn in figure $(b)$ correspond to those in figure $(a)$.

Thus, the replacement of the correlation function by a symmetrical function increases the redistribution term.

\section{Appendix C. Examples of space transformations}

The space transformation used in $\S 5.1$ is difficult to visualize in complex geometries. Furthermore, it is not straightforward to understand its use to solve a differential equation, for instance a Neumann problem, in a 3-dimensional geometry. This appendix aims to clarify these issues, by examples in 1- or 2-dimensional domains.

Figure 16 shows an example of such a transformation $\boldsymbol{\alpha}$ in the domain $[0,1] \times[0,1]$. The transformation is defined by:

$$
\left\{\begin{array}{l}
\alpha_{1}(x, y)=\sin ^{1 / 2}\left[\frac{\pi}{2}(0.05+0.9 y) x\right] / \sin ^{1 / 2}\left[\frac{\pi}{2}(0.05+0.9 y)\right] \\
\alpha_{2}(x, y)=\sin ^{2}\left[\frac{\pi}{2}(0.95-0.9 x) y\right] / \sin ^{2}\left[\frac{\pi}{2}(0.95-0.9 x)\right] .
\end{array}\right.
$$

This example may appear quite complicated, but it was chosen in such a way that the distortion of the domain is quite severe. This figure shows that the boundaries of the domain can be preserved even when no point of the domain is invariant, except for the corners.

However, the Green function of the domain $[0,1] \times[0,1]$ can only be obtained by applying a Fourier series expansion in one direction, which increases significantly the complexity of the analytic development. Therefore, in the remainder of this appendix, we will consider the case of a 1-dimensional Dirichlet problem:

$$
\left\{\begin{array}{l}
\nabla^{2} f(x)=g(x)=x, \\
f(0)=1, \\
f(1)=0 .
\end{array}\right.
$$

The Green function for the Dirichlet problem is

$$
\left\{\begin{array}{lll}
G\left(x, x^{\prime}\right)=(x-1) x^{\prime} & \text { for } & x^{\prime}<x \\
G\left(x, x^{\prime}\right)=\left(x^{\prime}-1\right) x & \text { for } \quad & x<x^{\prime}
\end{array}\right.
$$


The solution of (C 2) is then given by

$$
f(x)=\int_{0}^{x}(x-1) x^{\prime 2} \mathrm{~d} x^{\prime}+\int_{x}^{1} x\left(x^{\prime}-1\right) x^{\prime} \mathrm{d} x^{\prime}+1-x,
$$

and, finally,

$$
f(x)=\frac{x^{3}}{6}-\frac{7}{6} x+1
$$

The above is the direct way of solving (C 2) by means of the Green function (obviously not the simplest way to solve this problem). The problem can also be solved by introducing first a space transformation $\alpha$. For instance, let us consider the transformation

$$
\alpha:\left\{\begin{array}{ccc}
{[0,1]} & \longrightarrow & {[0,1]} \\
x & \longmapsto & x^{1 / 2} .
\end{array}\right.
$$

and the functions $\varphi=f \circ \alpha^{-1}$ and $\zeta=\partial^{2} \varphi / \partial x^{2}$. Now, $\zeta$, the Laplacian of $\varphi$, can be expressed as:

$$
\zeta(x)=\left(\frac{\partial^{2} f}{\partial x^{2}} \circ \alpha^{-1}\right)\left(\frac{\partial \alpha^{-1}}{\partial x}\right)^{2}+\left(\frac{\partial f}{\partial x} \circ \alpha^{-1}\right)\left(\frac{\partial^{2} \alpha^{-1}}{\partial x^{2}}\right),
$$

which yields

$$
\zeta(x)=5 x^{4}-\frac{7}{3}
$$

The function $\varphi$ is then the solution of the Dirichlet problem in $[0,1]$ :

$$
\left\{\begin{array}{l}
\nabla^{2} \varphi(x)=\zeta(x)=5 x^{4}-\frac{7}{3}, \\
\varphi(0)=1 \\
\varphi(1)=0 .
\end{array}\right.
$$

Since the domain has been preserved, the Green function is the same, and the solution of (C 9) is

$$
\varphi(x)=\int_{0}^{x}(x-1) x^{\prime}\left(5 x^{4}-\frac{7}{3}\right) \mathrm{d} x^{\prime}+\int_{x}^{1} x\left(x^{\prime}-1\right)\left(5 x^{4}-\frac{7}{3}\right) \mathrm{d} x^{\prime}+1-x,
$$

which leads to

$$
\varphi(x)=\frac{x^{6}}{6}-\frac{7}{6} x^{2}+1 .
$$

Finally, one can return to the original space using $f=\varphi \circ \alpha$, which yields (C 5) again.

This simple example shows how the functions are transformed and how one can obtain the same result by working in the transformed space. In principle, this method can be extended to multi-dimensional spaces. In complex geometries the method is obviously still valid, but the Green function cannot be determined analytically.

\section{REFERENCES}

Behnia, M., Parneix, S. \& Durbin, P. 1998 Prediction of heat transfer in a jet impinging on a flat plate. Intl J. Heat Mass Transfer 41, 1845-1855.

Bradshaw, P. 1973 The strategy of calculation methods for complex turbulent flows. Rep. 73-05, Dept of Aero., Imperial College, London. 
Bradshaw, P., Mansour, N. N. \& Piomelli, U. 1987 On local approximations of the pressure-strain term in turbulence models. Proc. Summer Program, CTR, Stanford University/NASA Ames Research Center, pp. 159-164.

Chou, P. Y. 1945 On velocity correlations and the solutions of the equations of turbulent fluctuation. Q. Appl. Maths 3, 38-54.

Craft, T. J. \& Launder, B. E. 1996 A Reynolds stress closure designed for complex geometries. Intl J. Heat and Fluid Flow 17, 245-254.

Demuren, A. O. \& Sarkar, S. 1993 Systematic study of Reynolds stress closure models in the computations of plane channel flows. J. Fluids Engng 115, 5-12.

Durbin, P. A. 1991 Near-wall turbulence closure modeling without "damping functions". Theoret. Comput. Fluid Dynamics 3, 1-13.

Durbin, P. A. 1993 A Reynolds stress model for near-wall turbulence. J. Fluid Mech. 249, 465-498.

Durbin, P. A. 1995 Separated flow computations with the $k-\varepsilon-\overline{v^{2}}$ model. AIAA J. 33, 659-664.

Durbin, P. A. \& Laurence, D. 1996 Nonlocal effects in single point closure. Proc. Turbulence Research Associates 96 meeting, Seoul, Korea.

Favre A. J., Gaviglio J. J. \& Dumas, R. J. 1957 Space-time double correlations and spectra in a turbulent boundary layer. J. Fluid Mech. 2, 313-342.

Favre A. J., Gaviglio J. J. \& Dumas, R. J. 1958 Further space-time correlations of velocity in a turbulent boundary layer. J. Fluid Mech. 3, 344-356.

Fu, S., LAunder, B. E. \& Tselepidakis, D. P. 1987 Accommodating the effects of high strain rates in modelling the pressure-strain correlation. Rep. TDF/87/5, Mech. Engng Dept, UMIST, Manchester.

Gibson, M. M. \& LAunder, B. E. 1978 Ground effects on pressure fluctuations in the atmospheric boundary layer. J. Fluid Mech. 86-3, 491-511.

Grant, H. L. 1958 The large eddies of turbulent motion. J. Fluid Mech. 4, 149-190.

HADžIĆ, I. 1999 Second-moment closure modelling of transitional and unsteady turbulent flows. PhD Thesis, Delft University of Technology.

Hanjalić, K. \& Launder, B. E. 1972 Fully developed asymmetric flow in a plane channel. $J$. Fluid Mech. 51, 301-335.

Hunt, J. C. R. \& Graham, J. M. R. 1978 Free-stream turbulence near plane boundaries. J. Fluid Mech. 84, 209-235.

Hunt, J. C. R., Moin, P., Lee, M., Moser, R. D., Spalart, P., Mansour, N. N., Kaimal, J. C. \& GaYnor, E. 1989 Cross correlation and length scales in turbulent flows near surfaces. Proc. 2nd Euro. Turb. Conf., 128-134.

KIM, J. 1989 On the structure of pressure fluctuations in simulated turbulent channel flow. $J$. Fluid Mech. 205, 421-451.

Laurence, D. \& Durbin, P. 1994 Modeling near wall effects in second moment closures by elliptic relaxation. Proc. Summer Program, CTR, Stanford University/NASA Ames Research Center.

Launder, B. E., Reece, G. J. \& Rodi, W. 1975 Progress in the development of a Reynoldsstress turbulence closure. J. Fluid Mech. 68, 537-566.

Launder, B. E. \& Tselepidakis, D. P. 1991 Progress and paradoxes in modelling near-wall turbulence. Proc. 8th Symp. Turbulence Shear Flows, Vol. 29-1, pp. 1-6.

Lien, F.-S., Durbin, P. A. \& PARneix, S. 1997 Non-linear $\overline{v^{2}}-f$ modelling with application to aerodynamic flows. Proc. 11th Symp. Turbulence Shear Flows, Vol. 6, pp. 19-24.

Lumley, J. L. 1975 Pressure-strain correlation. Phys. Fluids 18, p. 750.

Manceau, R. 1999 Modélisation de la turbulence. Prise en compte de l'influence des parois par relaxation elliptique. PhD Thesis, École Centrale de Nantes, France.

Manceau, R., Parneix, S. \& Laurence, D. 2000 Turbulent heat transfer predictions using the $\overline{v^{2}}-f$ model on unstructured meshes. Intl J. Heat and Fluid Flow 21, 320-328.

Manceau, R. \& Hanjalić, K. 2000 A new form of the elliptic relaxation equation to account for wall effects in RANS modelling. Phys. Fluids 12-9, 2345-2351.

Mansour, N. N., Kim, J. \& Moin, P. 1988 Reynolds-stress and dissipation-rate budgets in a turbulent channel flow. J. Fluid Mech. 194, 15-44. 
Monin, A. S. \& Yaglom, A. M. 1975 Statistical fluid mechanics. MIT Press.

Moser, R. D., Kim, J. \& Mansour, N. N. 1999 DNS of turbulent channel flow up to $R e_{\tau}=$ 590. Phys. Fluids, in press.

NaOt, D., Shavit, A. \& Wolfshtein, M. 1973 Two-point correlation model and the redistribution of Reynolds stresses. Phys. Fluids 16, 738-743.

Parneix, S., Durbin, P. A. \& Behnia, M. 1998 Computation of 3-D turbulent boundary layers using the V2F model. Flow, Turbulence and Combust. 60, 19-46.

Perot, J. B. \& Moin, P. 1993 Shear-free turbulent boundary layers: physics and modeling. Technical report, Dept Mech. Eng., Stanford University.

Rotta, J. C. 1951 Statistische Theorie nichthomogener Turbulenz. Z. Phys. 129, 547-572.

Sавот, J. 1976 Étude de la cohérence spatiale et temporelle de la turbulence établie en conduite circulaire. PhD Thesis, Lyon University, France.

Speziale, C. G. 1985 Modeling the pressure gradient-velocity correlation of turbulence. Phys. Fluids 28, 69-71.

Speziale, C. G., Sarkar, S. \& Gatski, T. B. 1991 Modeling the pressure-strain correlation of turbulence: an invariant dynamical system approach. J. Fluid Mech. 227, 245-272.

Wizman, V., Laurence, D., Kanniche, M., Durbin, P. \& Demuren, A. 1996 Modeling near-wall effects in second-moment closures by elliptic relaxation. Intl J. Heat and Fluid Flow 17, 255-266. 